\section{Histoire Épistémologie Langage}

43-2 | 2021

Linguistique psychologique et sémiotique : le contexte allemand et son influence

\title{
Zur Diffusion (kommunikations-)semantischer Modellgedanken aus dem Umkreis der deutschen Phänomenologie
}

Clemens Knobloch

\section{OpenEdition}

Journals

Édition électronique

URL : https://journals.openedition.org/hel/1082

DOI : $10.4000 /$ hel. 1082

ISSN : $1638-1580$

\section{Éditeur}

Société d'histoire et d'épistémologie des sciences du langage

Édition imprimée

Date de publication : 31 décembre 2021

Pagination : 83-112

ISBN : 9791091587150

ISSN : 0750-8069

\section{Référence électronique}

Clemens Knobloch, « Zur Diffusion (kommunikations-)semantischer Modellgedanken aus dem Umkreis der deutschen Phänomenologie », Histoire Épistémologie Langage [En ligne], 43-2 | 2021, mis en ligne le 01 décembre 2021, consulté le 17 décembre 2021. URL : http://journals.openedition.org/ hel/1082 ; DOI : https://doi.org/10.4000/hel.1082

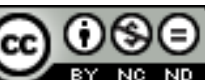

HEL is licensed under a Creative Commons Attribution-NonCommercial-NoDerivatives 4.0 International License 


\section{ZUR DIFFUSION (KOMMUNIKATIONS-)SEMANTISCHER MODELLGEDANKEN AUS DEM UMKREIS DER DEUTSCHEN PHÄNOMENOLOGIE}

\section{Clemens Knobloch}

Universität Siegen, Deutschland

\begin{abstract}
Zusammenfassung - Ausgehend von der Geschichte der semantischen Theoriebildung in den Jahren um 1900 verfolgt der Beitrag die rezeptionsgeschichtlichen Spuren, welche durch Phänomenologie, Brentanoschule und durch Heinrich Gomperz' Semasiologie veranlasst sind. Die zentrale These des Beitrags lautet: Mit Ogden \& Richards' The Meaning of Meaning (1923) sind die Weichen gestellt worden, die von der psychologistischen Vorstellungssemantik des 19. Jahrhunderts zur bis heute in der Sprachwissenschaft vorherrschenden Referenzsemantik führen. Alternative ,noetische" semantische Konzeptionen, Theorien des Gemeinten, sind in der linguistischen Semantik marginal und minoritär geblieben. Folgenreicher waren sie für Ethnolinguistik und Ethnomethodologie.
\end{abstract}

Stichwörter - Bedeutung, Referenz, Zeichendreieck, noetisch, Proposition, Intentionalität, Gemeintes, Psychologie, Situation

\begin{abstract}
Starting from the history of semantic theory around 1900 , this paper follows the traces left in semantics by the anti-psychologist networks of phenomenology, by the pupils of Franz Brentano, and by the Semasiology of Heinrich Gomperz. The central suggestion of the text is that Ogden \& Richards' The Meaning of Meaning (1923) paved the way toward transforming 19th century psychological semantics into modern referential semantics, still dominating linguistic thinking today. Alternative semantic theories based on "noetic" models of the thing-meant have remained marginal up to now, but have been more consequential in Ethnolinguistics and Ethnomethodology.
\end{abstract}

Keywords - meaning, reference, semiotic triangle, noetic, proposition, intentionality, thing-meant, psychology, situation

\section{VORAB}

Bedeutung und Referenz sind als konventionell-kanonische Grundbegriffe der Linguistik gewiss geeignete Kandidaten für Saussures notorische Klage, er kenne keinen einzigen sprachwissenschaftlichen Traditions- und Grundbegriff, der so präzise definiert wäre, dass sich mit ihm tatsächlich etwas anfangen ließe ${ }^{1}$.

In der semantischen Theoriebildung trifft um 1900 herum ein vorstellungspsychologischer mainstream (Hermann Paul z. B.) mit einer äußerst vielseitigen 
französischen, deutschen und englischen Szene zusammen, deren Mitglieder sich teils zeichentheoretisch, teils psychologisch, teils philosophisch, teils auch kommunikationswissenschaftlich verstehen (vgl. Nerlich 1992: 247-256). Am (vorläufigen) Ende dieser Begegnung steht mit Ogden \& Richards (1923) das vielleicht einflussreichste, jedenfalls aber das wirrste Semantikkompendium des 20. Jahrhunderts. Es erlebt mehr als 10 Auflagen und wird in zahlreiche Sprachen übersetzt. Lesen muss man es - so meine These - als Zeugnis einer Regression. Alle Versuche, die Grenzen des aristotelischen Zeichendreiecks (Laut- oder Schriftzeichen - Konzept/Bedeutung/Inhalt - Sache/Referent) im ersten Jahrhundertdrittel zu sprengen, münden da just wieder an ihrem Ausgangspunkt ${ }^{2}$.

$\mathrm{Zu}$ den eher philosophischen Autoren, die um 1900 aus der aristotelischen Gefangenschaft auszubrechen versuchen, gehören in Deutschland Edmund Husserl, Anton Marty, Heinrich Gomperz und einige andere. Sie sind alle bei Ogden \& Richards rezipiert (und im Appendix der amerikanischen Ausgabe finden sich Exzerpte und Notizen zu ihnen), aber sie kommen nur später und überwiegend bei fachlichen Außenseitern zu ihrem Recht ${ }^{3}$. Von Ludwik Fleck (1980 [1935]), dem Begründer der historischen Wissenschaftssoziologie, wissen wir, dass auch wissenschaftliche Inhalte gar nicht anders können als sich zu verändern, wenn sie zirkulieren, gleich ob in der fachlichen Eigengruppe oder in einer Fremdgruppe. Wir dürfen also nicht mit stabilen Wissensbeständen rechnen, sondern mit jeweils anders nuancierten und ausgelegten heuristischen Konzepten.

Im folgenden Text geht es um „alternative“ Semantikkonzeptionen, die wesentliche Anregungen aus dem Umfeld der Brentanoschule ${ }^{4}$ und der (weit verstandenen) Phänomenologie erfahren haben. Es mag vermessen erscheinen, wenn ich hier auch Heinrich Gomperz, einen Schüler Ernst Machs, dem Umfeld der Phänomenologie zurechne (wohlgemerkt: dem Umfeld, nicht der Phänomenologie selbst). Neben der in Gomperz $(1908,1929)$ immer wieder geführten Auseinandersetzung mit Husserl spricht dafür, dass es auch Stimmen gibt, die Machs Version des „Positivismus“ als einen partiell affinen Vorläufer der Phänomenologie betrachten (z. B. Lübbe 1960). Dafür spricht weiterhin, dass kein Geringerer als Bühler (1927: 4) auf Affinitäten zwischen Mach und der Brentanoschule hinweist. Gomperz' „Noologie“, das sollte freilich festgehalten werden, hat in der (von ihm alsbald wieder aufgegebenen) „Weltanschauungslehre" die Aufgabe, logische Grundbegriffe auf ihre Verträglichkeit mit den korrelativen Prozessbegriffen der Psychologie hin zu kalibrieren.

Es geht im Folgenden keinesfalls um die (richtige oder falsche) Auslegung der Quellentexte von Husserl und Gomperz. Ein Beitrag zur Gomperz- oder gar zur Husserl-Philologie ist nicht beabsichtigt. Geschrieben ist der Text aus der

\footnotetext{
$2 \mathrm{Zu}$ den Ausgaben, Einflüssen und Schicksalen von The Meaning of Meaning vgl. Gordon (1994). Das Verhältnis zu Russell und den Logischen Atomisten rekonstruiert McElvenny (2014).

3 Die philologische Dignität dieser „Rezeption“ bei Ogden \& Richards lässt sich freilich daran ermessen, dass Gomperz (1908) dort nach einer sekundären Quelle referiert wird, nämlich nach Dittrich (1913). Und Ottmar Dittrich war der sprachpsychologische Schüler Wilhelm Wundts und ein wissenschaftlicher Gegner von Gomperz. Vgl. hierzu auch Kiesow (1988).

4 Zur Spannweite der Brentanoschule vgl. Fréchette (2018).
} 
Perspektive derjenigen Semiotiker und Semantiker, die sich in der Theorie und Gedankenwelt von Gomperz und Husserl (sagen wir) selektiv bedient haben.

Was Gomperz betrifft, dürften die rezeptionsgeschichtlich bekannten Wirkungen begrenzt sein auf das berühmte Zeichendreieck bei Ogden \& Richards (1923), auf das ich gleich zurückkomme, auf Bühlers ausführliche Befassung mit ihm in der Sprachtheorie (Bühler 1934: 40 ff.) sowie auf Karl Popper und den Wiener Kreis (vgl. z. B. Kiesow 1988, Seiler \& Stadler 1994) ${ }^{5}$. Wesentlich schwerer einzugrenzen sind die Anregungen, die aus der Brentanoschule stammen, zumal sie in der Psychologie mehr oder weniger übergangslos mit dem gesamten Gestalt-Komplex verbunden sind (Carl Stumpf und seine Berliner Schüler, Alexius Meinong in Graz etc.). Auch reichen ihre Wirkungen weit über Psychologie und (sprachwissenschaftliche) Semantik hinaus in den Bereich der (entstehenden) Kultur-, Sozial- und Kommunikationswissenschaften hinein. Der Psychologe Karl Bühler (1927) war ja keinesfalls der einzige, der sich in den 1920er Jahren mit Zeichenverkehr und kulturellen Objektivationen befasste. Eher kultursoziologisch verstanden sich in dieser Zeit Autoren wie Hans Freyer (1923) - breit rezipiert in den Arbeiten Bühlers und auch bei Gomperz (1929) selbst später diskutiert - oder in den USA Florian Znaniecki (1919) oder die frühen (ebenfalls kommunikations- und zeichentheoretischen) Arbeiten von Karl Mannheim (vgl. Maas 2019) .

In der deutschsprachigen akademischen Sprachwissenschaft finden wir darüber hinaus ein spätes Echo des phänomenologischen Umkreises bei Autoren wie Porzig (1950), Sandmann (1940, 1954), Nehring (1963), Koschmieder (1965), Hempel (1980) ${ }^{7}$. Eine minimale Klammer für all diese aus dem Umkreis der Phänomenologie inspirierten Semantiken besteht darin, dass sie alle Axiomatik und Denkstil der vorstellungspsychologischen Semantik (vgl. hierzu Knobloch 1988: 239-321) in einer Richtung verlassen, die nicht zur Referenz (wie bei Ogden \& Richards 1923), sondern zu intentionalen Gegenständen, zu einer Theorie des Gemeinten (im weiten Sinne) führen.

5 Soweit ich sehe, hat niemand auch nur versucht, Gomperz' ,pathempirischen“ Systemgedanken aufzunehmen (vgl. aber jetzt Romand 2019). Den Versuch der Fundierung aller intellektuellen Leistungen in affektiven Komplexen (so Kiesow 1988: 349 f.) sah Gomperz selbst alsbald für unzureichend an. In einem posthum veröffentlichten kurzen autobiographischen Text schreibt er: „I was still young when I endeavored to work out a system of philosophy based on the assumption that the functions mostly considered as specifically rational were in fact emotional. This basis was clearly too narrow and when two volumes had been published I abandoned the undertaking“ (Gomperz 1953: 21). Popper hingegen nutzt das Zeichenmodell Gomperz' für sein Falsifikationsprinzip. Die Rezeption ist durchweg sehr selektiv.

6 Hans Freyer gilt als Begründer der Leipziger Schule der deutschen Soziologie, aus der auch Arnold Gehlen und Helmut Schelsky hervorgegangen sind. Er war ein aktiver Parteigänger des Nationalsozialismus. Seine Theorie des objektiven Geistes (Freyer 1923) analysiert kulturelle Gebilde, Zeichen und Sozialformen als objektivierte Vorgaben gesellschaftlicher Kommunikation und ist (nicht nur, aber auch über Karl Bühler) für die moderne Kommunikationssoziologie bedeutsam geworden. Florian Znaniecki gilt als Begründer der polnischen Kultursoziologie, ist aber vor allem über seine US-amerikanischen Veröffentlichungen (vor allem zusammen mit W. I. Thomas) wirksam geworden und zählt zu den Anregern der ,ethnomethodologischen“ Kommunikationssoziologie Harold Garfinkels (vgl. Garfinkel 2006 [1948]).

7 Vgl. hierzu auch Maas (2016: 350 ff., 380 ff.; 2020), besonders zu Porzig wie auch zur hier weitgehend vernachlässigten Husserlrezeption bei Roman Jakobson und den Prager Strukturalisten (vgl. Holenstein 1975 und Albano Leoni 2015). 


\section{NOETIK UND INTENTIONALITÄT}

Es ist nicht ganz unkompliziert, die Grund- und Leitbegriffe, um die es hier geht, zielführend zu definieren. Ich beginne mit Bühlers Lesart von „Intentionalität“"

Es ist das unvergängliche Verdienst Franz Brentanos, das Merkmal der Intentionalität, des Hinweisens, Gerichtet-, Bezogenseins der Erlebnisse [...] begrifflich scharf erfasst und gebührend gewürdigt zu haben. Die punktmäßige, sozusagen statische Intentionalität jedes Einzelerlebnisses für sich betrachtet wäre eine Kuriosität; wenn wir darin aber mehr, nämlich eine dynamische, eine Ablaufszuordnung erblicken, dann gewinnt die Intention die natürliche Funktion eines Steuerhebels, durch den Erlebnisse in uns in Konkordanz mit den Struktur- und Daseinsgesetzen der Gegenstände gebracht werden können. (Bühler 1927: 67)

Diese (in ihrem sorglosen Ungefähr durchaus Bühler-typische) Definition erlaubt im Kontext der Krise der Psychologie von 1927 vielleicht den Schluss, dass die Intentionalität unseres Erlebens ein Mittel zur Objektivierung und zur Sozialisierung unserer Bewusstseinsvorgänge sei. Schließlich geht es um die gegenseitige Steuerung des sinnvollen Benehmens von Gemeinschaftsmitgliedern, und da wäre jede subjektive und individuelle Form von bewusstem Erleben anomisch und disfunktional. Die Intentionalität der Brentanoschule ist nicht bedeutungsgleich mit dem Alltagsausdruck gleichen Namens, der auf eine (zugeschriebene) Sprecher- bzw. Akteursabsicht verweist. Es geht vielmehr um die inhärente Gerichtetheit aller (und zumal der zeichenhaften) psychischen Prozesse auf etwas, das außerhalb von ihnen verortet und tendenziell sozial objektiviert werden muss. Was die konventionelle Semantik als Referenz bezeichnet, das bildet (so gesehen) einen Grenzfall von Intentionalität, bei welchem der intentionale Gegenstand zudem auch noch perzeptiv eingelöst werden kann in einem singulären Objekt ,da draußen“. Die Intentionalität ist dann „erfüllt“", sie wird „bündig“ in der geteilten Wahrnehmungs- und Handlungssituation $^{8}$. Charakteristisch für den Gebrauch von Sprachzeichen ist aber, dass ihre intentionalen Gegenstände uns auch dann (als objektiviert und sozialisiert) gegeben sind, wenn sie nicht perzeptiv erfüllt (oder überhaupt erfüllbar) sind. Es genügt, wenn sie auf geteilte Erfahrungen und Erwartungen verweisen, schon dadurch etablieren sie „Zentren möglicher Ausgiebigkeit" ${ }^{\text {"9 }}$, von denen aus weiter expliziert werden kann, was in und mit ihnen intendiert ist.

Auch Karl Mannheims zeitgleiche kultursoziologische Reflexionen über konjunktives und kommunikatives Denken (Mannheim 1980 [1924]: 155-322) ${ }^{10}$ weisen in die gleiche Richtung (vgl. Maas 2019). Eigentlich stehen die sprachlichen Ressourcen allen Teilnehmern gleichermaßen zur Verfügung (,im Prinzip"), andererseits muss der Grad der Übereinstimmung in der kommunikativen Praxis beständig präzisiert und überprüft werden. Die stabilen Gemeinsamkeiten, die sprachliche Ausdrücke in der noetischen Zuwendung zu präsentieren scheinen, stehen lediglich für eine Schnittstelle (heute würde man wohl von

8 Die Formel von der „Bündigkeit“ der zeichenhaften Steuerungen übernimmt Bühler von Freyer (1923).

9 Um eine Formulierung von Arnold Gehlen zu verwenden.

10 Die Angaben zur Entstehung des Manuskripts schwanken zwischen 1922 und 1924. 
„boundary objects“ sprechen), an der sich die Teilnehmer der Kommunikation begegnen und austauschen.

Anders gesagt: Jede sprachliche Äußerung ruft so etwas auf wie einen referentiellen Horizont, gleich ob er perzeptiv erfüllt werden kann oder nicht (das ist bedeutsam für Gomperz' Zeichenmodell, wie gleich zu zeigen sein wird). Und weiterhin können auch explizit rahmende sprachliche Ausdrücke selbst Verweishorizonte aufrufen, in denen andere, folgende Ausdrücke intentional und indexikalisch bündig werden. Man denke an Überschriften (,entitlements“ bei Kenneth Burke), die als explizite Rahmungen (und post hoc als Zusammenfassungen) des sequentiellen Sprachgeschehens dienen. Dabei gilt, dass die explizite Rahmung auf ihre eigene indexikalische Fundierung verweist und umgekehrt alle Formen der prädikativen Explikation indexikalisch fundiert sind ${ }^{11}$.

Dieser Umstand bringt uns direkt zur Wortfamilie Noesis, Noema, Noetik, noetisch. Sie handelt (in der phänomenologischen Tradition) von der Bewusstseinsgegebenheit der Verstandesgegenstände. Noemata stehen für deren ,gegenständlichen“"Kern (wobei „gegenständlich“ hier eben nicht perzeptiv erfüllt heißt) zusammen mit seinen wechselnden Gegebenheitsweisen. $\mathrm{Zu}$ diesen gehört dann u. a. auch die perzeptiv-anschauliche Gegebenheit, aber eben auch die in der Erinnerung oder im Abbild. Jeder noematische Inhalt ist doppelt, einerseits selbst notwendig Prädikat und Bestimmung, andererseits auch weiter thetisch explizierbar. Das hat eine ungefähre strukturelle Entsprechung in der SubjektPrädikat-Opposition des Satzes und in der Verteilung der Hauptwortarten auf nennend-identifizierende, modifizierende und (prädikativ) charakterisierende Funktionen. Insofern sie die Denknotwendigkeiten der sprachlichen Darstellung berücksichtigen müssen, sind die grammatischen Kategorien der Einzelsprachen noetisch fundiert.

Noesis und Noema gehören zu jedem intentionalen Erlebnis: Bewusstsein von etwas und vermittelt durch etwas als Einheit. Neben dem intendierten Gegenstand gibt es in Husserls Variante der Noetik die Variable der Aktqualitäten. Sie steht für die in der Brentanoschule mit vielen Varianten gepflegte Ontologie der Bewusstseinsinhalte, die uns gegeben sein können im (heute würde man sagen: kognitiven) Modus ihrer Wasbestimmtheit, im affektiven Modus des (un-) erwünschten, im problematisierenden, im konditionalen Modus etc. ${ }^{12}$ Zur Vereinfachung des Verständnisses: Die Sphäre der Aktqualitäten entspricht ungefähr der pragmatisch-kommunikativen, modalisierenden Vollzugsrealität des Sprechens, der konstant gesetzte noematische Kern entspricht ungefähr der kognitivsemantischen Perspektive der sekundären Reflexion. Für beide Seiten gilt allerdings, dass sie nicht in der alltäglichen Vollzugsrealität des Sprechens, in der Praxis, sondern eben erst in der reflexiven Zuwendung in ihrem noetischen Charakter zugänglich werden, wenn die „natürliche Einstellung“ der alltäglichen

11 Was die (hier keineswegs beabsichtigte) philologische Rekonstruktion der Lesarten von „Intentionalität“ in der Brentanoschule betrifft, so unterscheidet Fréchette (2018: 107 ff.) allein bei Bühler mindestens acht unterschiedliche „Versionen“ des Begriffes.

12 Bei Marty (1908) steht an dieser systematischen Stelle die Dreiteilung der Sprachmittel in Vorstellungssuggestive, Emotive und interesseheischende. 
Praxis eingeklammert wird ${ }^{13}$. Noetisch heißt bei Husserl (zumal in Husserl 1913 [1901]) die Perspektive, in der die Bedeutungen als reine Verstandesgegenstände thematisiert werden ${ }^{14}$. Noetische Kategorien sind so konstitutiv verbunden mit allen Formen sprachlicher Praxis, sie finden aber ihre empirisch-kategorialen Ausdrucksformen in den historischen Gegebenheiten der Einzelsprachen. Hierin liegt die Affinität der Phänomenologie zu einer zeitgemäßen Erneuerung der „Allgemeinen Grammatik“ (insbesondere bei Marty 1908, aber auch bei Husserl). Gomperz teilt diese Affinität nicht.

Kommen wir zur Verwendung des Adjektivs „noëtisch“ bei Gomperz ${ }^{15}$. Sie unterscheidet sich in wenigen Punkten von der phänomenologischen, die durchweg mit Affinitäten zu Gomperz' Lehrer Ernst Mach zusammenhängen. „Noëtische Gegenstände“ sind (vgl. Gomperz 1908: 102) definiert als die Einheit von Aussageinhalt und Aussagelaut, konstituiert gewissermaßen durch das bilaterale komplexe Sprachzeichen allein, unter expliziter Nichtbetrachtung der jeweiligen Aussagegrundlage (vgl. das auf S. 92 reproduzierte Doppeldreieck). Diese letztere nämlich gehört im Entwurf zu Gomperz' „Weltanschauungslehre“ nicht zur semasiologischen, sondern zur ontologischen Betrachtung. Die solchermaßen bestimmten noëtischen Komplexe sind einesteils selbständige, autonome (und für sich in wechselnden Lebenslagen reproduzierbar und rekombinierbar), in der jeweiligen Aussagepraxis treten sie in fallweise spezifische typisierende Beziehung zu der Aussagegrundlage, mit der sie gekoppelt sind. Diese Kopplung freilich bewerkstelligt Gomperz mit der (einigermaßen überraschenden) These, es sei der nämliche Komplex „logischer Gefühle“, der den noetischen Sinn der Aussage und die Auffassung der Aussagegrundlage zusammenhalte (ibid.: 279) ${ }^{16}$. Dass Gomperz mit einer solchen, unter keinen denkbaren Bedingungen falsifizierbaren Annahme zum Gewährsmann des Popper'schen Falsifikationismus avancieren konnte, entbehrt gewiss nicht der geistesgeschichtlichen Ironie. In seinem späteren Werk spielt dieser Komplex indes keine Rolle mehr. Die ungewöhnliche Kopplung von Logik und Gefühl steht hier für den Versuch, mit der dualistischen (Logik und Psychologie versöhnenden) Axiomatik Ernst Machs kompatibel zu bleiben.

Alle anderen Verwendungen des Adjektivs „,noëtisch“ bei Gomperz (ibid.) sind direkt verbunden mit diesem ,pathempirischen“ Projekt der Weltanschauungslehre des frühen Gomperz' und entstammen den Systemzwängen, welche durch die Vereinbarkeit mit Mach in sein System importiert werden. Schließlich müssen die semiologische und die ontologische Sphäre versöhnt

13 Für diese Definitionsversuche stütze ich mich auf Maas (2020) sowie auf die einschlägigen Einträge in Ritter \& Gründer (1984).

14 Als typische noetische Gegenstände gelten darum bei phänomenologisch geprägten Semantikern die Subjektsausdrücke theoretisch erklärender, definierender, mathematischer Sätze.

$15 \mathrm{Zu}$ den orthographischen Marotten Gomperz' gehört es, dass er den Ausdruck stets mit Doppelpunkt-e schreibt.

16 „Aussage und Sachverhalt, noëtische und typische Gegenstände besitzen somit die gleiche Substanz: es ist derselbe Komplex logischer Gefühle, der den Worten ihren Sinn und den Tatsachen ihre Auffassung verleiht, und der dadurch jene zur Einheit einer Aussage, diese zur Einheit eines Sachverhalts zusammenfaßt“ (Gomperz 1908: 279). Zu Gomperz’ Lehre von den „logischen Formalgefühlen“ vgl. jetzt Romand (2019). 
werden. Der Gomperz von 1929 hat sich aus diesen Systemzwängen weitestgehend gelöst. Er steht Husserl näher als der (gleichnamige) Autor von 1908 und bewegt sich im US-Exil nach 1934 auf den logischen Positivismus zu. Bei den kultur- und kommunikationswissenschaftlichen Rezipienten der Debatte bleibt vom „pathempirischen“ Themenkomplex nichts. Bei den linguistischen Semantikern hinterlässt er ebenfalls keine Spur. In Bühler (1934) wird Gomperz (1908) verschiedentlich erwähnt und diskutiert, aber als Semiologe, nicht als Pathempiriker. Seine Sprachtheorie steht in der Hauptsache dafür, dass es für die Axiomatisierung dessen, was sprachliche Darstellung ausmacht, einen problemgeschichtlichen Rahmen gibt, der Semantik nicht als Referenz, sondern als einen Horizont des Gemeinten versteht. Differenzen zwischen den Modellen von Gomperz und Husserl gibt es zweifellos, und sie beginnen vielleicht da, wo das Gemeinte als situativ/perzeptiv, als okkasionell „erfüllt“", in das Bezeichnete übergeht, wo Gomperz seine Aussagegrundlage einer anderen (ontologischen) Ordnung zurechnet, wo Husserl die Vollzugsrealitäten der natürlichen Einstellung für seine Zwecke einklammert - und wo Bühler zu der (diese Differenzen elegant zudeckenden) Formel „Gegenstände und Sachverhalte“ greift. Diese Formel nämlich deckt Ontologisierungsbedürfnisse ebenso ab wie Semiotisierungsbedürfnisse. Sie bleibt neutral gegenüber der Frage, ob die sprachliche Darstellung (bildlich gesprochen) bei sich bleibt, wenn sie „Gegenstände und Sachverhalte" intentional meint, oder ob sie sich quasi-referenziell auf Außersprachliches bezieht.

Ich werde versuchen, den (hier nur vage umgrenzten) noetischen Strang im semantischen Modelldenken der Zeit um 1900 freizulegen, mit Schwerpunkt auf Gomperz (1908) und auf Husserl (1913 [1901]), mit einigen Randbemerkungen zu Husserl (1948 [1938]) - und darauf, was qua Wissenstransfer aus diesen Modellgedanken in der semantischen Theorie des 20. Jahrhunderts geworden ist. Insbesondere geht es auch um die Transformationen und Reformulierungen, die eher logisch-philosophische Modelle auf dem Weg zu einer Linguistik des Sprechens durchlaufen.

\section{Der Modellgedanke}

Eigentlich ist der elementare Modellgedanke für die Noetik ganz einfach. Er besagt, dass sprachliche Ausdrücke im tatsächlichen Gebrauch auf etwas ausgerichtet sind, was

[a] nicht sie selbst sein können (wir sprechen nicht über die Sprache, sondern mit ihr); was

[b] nicht ein subjektiver Bewusstseinsinhalt, eine Vorstellung im Sinne der sensualistischen Psychologie des 19. Jahrhunderts sein kann; und was

[c] nicht die Dinge der wirklichen Welt da draußen sein können, obwohl es deren Objektivität (und Intersubjektivität) aufzuweisen scheint. Dass wir buchstäblich über alles so sprechen können, als ob es die sachliche Objektivität von Häusern, Bäumen und Hunden hätte, ist dann doch ein zu auffälliger Zug unserer Sprachfähigkeit. 
Dabei ist es gleichgültig, ob der Sprecher für sich selbst oder für andere spricht. Für Husserl sind es die Stufen der sprachlichen Artikulation des Gemeinten, die zur noetischen Grundstruktur der Prädikation hinführen. Sie führen von der Demonstration mit okkasioneller Erfüllung über deren Anreicherung mit typisierenden Konzepten bis zur Artikulation des Prädikativen selbst (vgl. Maas 2020). Angelegt sind in diesen Artikulationsstufen die noetischen Modelle der Nominalität (Form der Bestimmbarkeit), der Verbalität (Form der Prädikation) und der Adjektivität (Form der modifizierenden Bestimmung).

Ad [a]: Dass der sprachsystemische Eigenwert ein hoch variables Werkzeug des Sprechens, aber grundsätzlich nicht die Zielgröße der Intentionalität von Ausdrücken ist, haben in der französischen Diskussion Michel Bréal (1897) und in der deutschsprachigen Karl Otto Erdmann (1922 [1900]) sehr stark gemacht. Erdmann spricht vom „Lob des gedankenlosen Wortgebrauchs“, offenbar in der Absicht, das 19. Jahrhundert zu provozieren, das gerne im Etymon eines Wortes dessen eigentliche und ursprünglich gemeinte Bedeutung sehen wollte! Für Erdmann und Bréal erfüllt das Wort erst dann seine kognitive (würden wir heute sagen) Funktion, wenn es selbst mit seinem Eigenwert hinter der fallweisen Verweisungsleistung zurücktritt, wenn es gewissermaßen verschwindet, medial und durchsichtig wird. Zwar wandert die Aufmerksamkeit beim Sprechen zwischen Bedeutung und gemeintem Gegenstand, die Bedeutung tritt aber in den Hintergrund, sobald sie den Weg zum Gemeinten gebahnt hat, sie ist ein Mittel, das aber als ,eigensinnig“ nie ganz verschwindet im intentional Angezielten (und auch selbst thematisiert werden kann) ${ }^{17}$. Es sind die Reste der Vorstellungs- und der Etymonsemantik des 19. Jahrhunderts, deren Auflösung man hier beobachten kann. Erdmann (ibid.: $162 \mathrm{f}$.) schreibt, es seien zufällige, unwesentliche, nebensächliche Merkmale, nach denen die Begriffe benannt werden. Für den Sprachwissenschaftler seien sie vielleicht von Interesse, für den Sprachbenutzer nicht.

Erst in der philosophischen oder sprachtheoretischen Reflexion werden die Bedeutungen selbst zu Gegenständen, auf die intentionale Akte sich richten können.

Auch Saussure hat diesen Zusammenhang genauestens seziert, wie wir zwar nicht aus dem kanonischen Text des CLG, aber aus den Nachlassanalysen von Fehr (1997) wissen. Der systemische Eigenwert, Saussures valeur eines Sprachzeichens ist die Seite, die dem darstellungstechnischen System der Einzelsprache zugewandt ist und gerade nicht dem Gemeinten.

Ad [b]: Dass das Gemeinte eines Sprachzeichens im Gebrauch kein individueller Bewusstseinsinhalt, keine Vorstellung (des Sprechers oder Hörers) sein kann, ist eine Pointe des Antipsychologismus in der Brentanoschule und besonders bei Husserl. Der nämlich zeigt immer wieder am Beispiel logischer Ausdrücke wie die Zahl vier, ein gleichschenkliges Dreieck etc. deren bewusstseinsunabhängige Objektivität. Das, worauf die intentio eines Sprachzeichens im Gebrauch geht, hat eine eigene Objektivität, die sich weder in der Sphäre des 
individuellen Erlebens, im Innenraum des Bewusstseins auflösen lässt noch in der nominalistischen Welt der Gegenstände da draußen ${ }^{18}$.

Ad [c]: Die nämlich können auch nicht als Garant der Objektivität des Gemeinten herangezogen werden, auf die sprachliche Ausdrücke verweisen. Wir können über Fiktionen, hypothetische Objekte, erfundene Textreferenten etc. genau so mühelos sprechen wie über unsere Nachbarn. Die idealen Gegenstände der Noetik, die wir beim Sprechen gebrauchen, haben hingegen wahrhafte Existenz, schreibt Husserl (1913 [1901]: 124-126). Zu denken wäre hier auch an Saussures mäandernde Gedanken über die Frage, was sich eigentlich hinter dem kontinuierlichen Gebrauch eines mythischen Götternamens wie Zeus (oder hinter den Namen der Gestalten aus der Nibelungensage) verbirgt. Es kann ja offenkundig in einem solchen Falle nicht der Namensträger sein, der die Identität des Zeichens verbürgt - wenn es ihn nie gegeben hat. Ironischerweise ist Fehrs (1997) Saussure-Buch selbst ein Beleg für die These Saussures, sofern es nämlich zeigt, dass man den kanonischen Saussure und den gleichnamigen Autor der nachgelassenen Notizen kaum als ein und denselben Referenten auffassen kann.

Objekt-Name-Komplexe, so heißt es in den Notizen Saussures, werden nicht in der Zirkulation der Zeichen weitergegeben, darum kann die Bezeichnungsrelation keine Basis für einen angemessenen Zeichenbegriff liefern. Das ist, wie Fehr (ibid.: 140) notiert, kritisch gegen Frege gerichtet.

Es ist eine ganz eigentümlich objektive Welt des Gemeinten, auf welche sprachliche Ausdrücke uns verweisen, und für deren Explikation bürgert sich bei einigen Autoren um 1900 der Name „noetisch“ ein. Noetische Kategorien und Dimensionen sind konstitutiv für alle Sprachen, ihre empirische Ausgestaltung erfolgt in der Einzelsprache. Bühlers (1934) Gegenstände-und-SachverhalteFormel aus dem Organonmodell meint eben nicht die rohe Außenwelt, er meint diejenige Seite der Zeichen im Gebrauch, die der noetischen Welt des Gemeinten zugewandt ist. In diesem Sinne hat er die Formel von Gomperz (1908: 73) übernommen, und auch bei Husserl findet man Entsprechendes (vgl. weiter unten). Der Unterschied ist wesentlich. Denn während Ogden \& Richards (1923: 115; in der deutschen Übersetzung 1974: 136) ganz treuherzig versichern, alle sprachlichen Bedeutungsprobleme ließen sich in Luft auflösen, wenn man nur von zweifelsfrei gemeinsamen Referenten, d.h. von evidenten Objekt-NameKomplexen ausginge, ergibt sich aus der Perspektive von Gomperz und Husserl ein völlig anderes (neues und wirkmächtiges) Bild des Bedeutungsproblems, eines, das die Besonderheiten der Sprache berücksichtigt und nicht in der uralten Tradition der aristotelischen Vermengung von Sprach- und Erkenntnisproblem stecken bleibt ${ }^{19}$. Die historische Beharrungskraft des aristotelischen Zeichendreiecks hat etwas Bestürzendes.

18 „Es ist ein Zufall, wenn das Zeichen einem Objekt entspricht, das definiert ist für die Sinne wie ein Pferd, das Feuer, die Sonne [...]“ zitiert Fehr (1997: 139) aus den Notizen Saussures. Und weiter: „Wenn ein Objekt, wo es auch sei, der Endpunkt sein könnte, auf den ein Zeichen fixiert ist, würde die Linguistik augenblicklich aufhören, das zu sein, was sie ist" (ibid.: 140). Auf die diesbezüglichen Affinitäten zwischen Husserl und Saussure verweist Maas (2020).

19 In der Tat hat ja die spätere Historiographie sowohl Gomperz (1908) als auch Ogden \& Richards (1923) für die Vorgeschichte des logischen Positivismus' des Wiener Kreises in Anspruch genommen, mit dem er 


\section{EINZELHEITEN ZUM BEDEUTUNGSPROBLEM: GOMPERZ VS. HUSSERL}

Während Husserl ${ }^{20}$ nicht eingeführt werden muss, ist Heinrich Gomperz (1908), für den ich historiographisch ein gutes Wort einlegen möchte, heute weitgehend unbekannt. Der Sohn des renommierten Wiener Gräzisten Theodor Gomperz war als Philosoph ein Schüler Ernst Machs. Nach der austrofaschistischen Machtergreifung wurde er zwangsemeritiert und emigrierte 1935 in die USA, wo er durch Vermittlung von F. C. S. Schiller eine Gastprofessur an der University of Southern California erhielt, auf der er bis zu seinem Tod 1942 lehrte (vgl. Maas 2018).

Gomperz” „Noologie“ von 1908 (nur der erste Teil ist erschienen) gehört in den Umkreis des Antipsychologismus der Zeit - insofern man darunter eben die Erkenntnis und Einsicht versteht, dass die „Gegenstände“ der Logik, des Denkens und der Wahrnehmung in den Prozessen des Denkens und des Wahrnehmens nicht aufgehen und diesen nicht eingeordnet werden können (vgl. hierzu die Diskussion in Gomperz 1929: 141 ff.).

Die Aufgabe der Disziplin „Noologie“ ist es laut Gomperz, die Widersprüche zwischen dem logischen und dem psychologischen Zugang zum Denken auszugleichen. Und das versucht Gomperz (1908) anhand einer ausgreifenden, als „Semasiologie" betitelten Entfaltung des Bedeutungsproblems. Wir nähern uns seiner Version des Bedeutungsproblems am besten, indem wir seine Version des semiotischen Dreiecks (ibid: 77 ) betrachten, die (so die communis opinio) Ogden \& Richards (1923) inspiriert haben soll und die vielen lediglich als Notationsvariante des heute klassischen Dreiecks gilt. Dem sieht sie auf den ersten Blick durchaus ähnlich. Erst der zweite Blick zeigt, dass wir es mit weit mehr als einer kosmetisch aufgehübschten Fassung des kanonischen Zeichendreiecks zu tun haben.

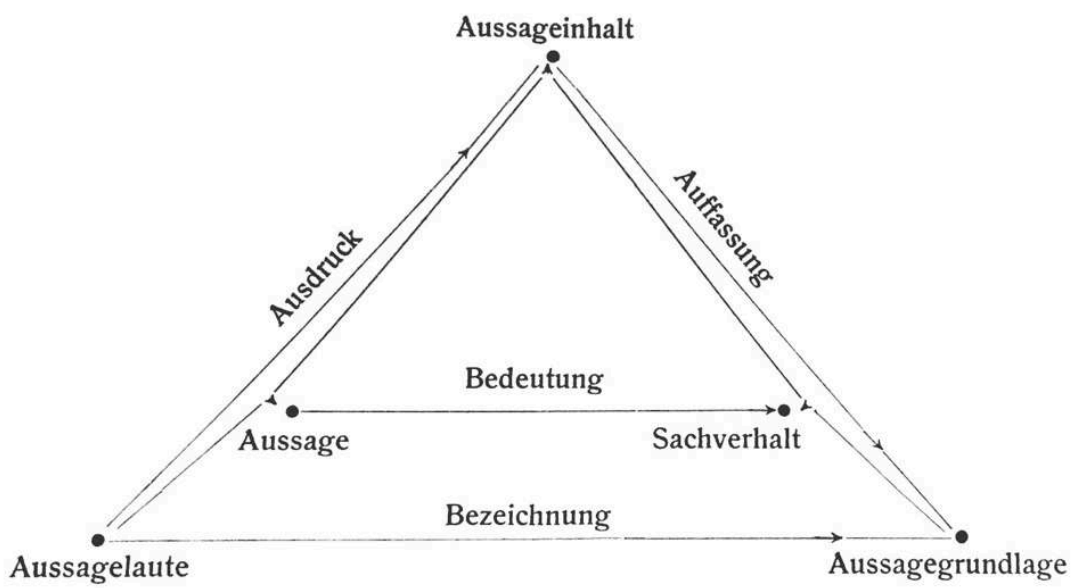

Abbildung 1: Das semiotische Dreieck aus H. Gomperz (1908: 77).

in den 20er Jahren und vor allem später im US-Exil Kontakt hatte; vgl. Seiler \& Stadler (1994), McElvenny (2014). In Gomperz (1929: 59-61, 141 ff.) überwiegt die Thematisierung von Differenzen zwischen Gomperz und den beiden kurz zuvor erschienen Werken Carnaps (Der logische Aufbau der Welt, Scheinprobleme der Philosophie).

20 Eine konzise Darstellung der Wirkung und Bedeutung Husserls für die Sprachtheorie gibt Maas (2020). 
Mit diesem teilt es die Relationsfundamente: Aussagelaute - Aussageinhalt Aussagegrundlage, was den Betrachter verführt, sich zu sagen: „Ach ja, das ist das gleiche wie Zeichen - Begriff - Ding oder wie Symbol - Konzept - Referent, kennen wir." Zu den drei primären Relationen: [a] Ausdruck (= Relation zwischen Aussagelauten und Aussageinhalt); [b] Auffassung (= Relation zwischen Aussageinhalt und Aussagegrundlage); [c] Bezeichnung (= Relation zwischen Aussagelauten und Aussagegrundlage) kommen indes hier bei Gomperz (1908: 77) zwei entscheidende Neuerungen, manifest in der Verdoppelung des Dreiecks und in dem Umstand, dass eine vierte „Relation zwischen zwei Relationen“ eingeführt ist. Sie ist es, die den Namen „Bedeutung“ trägt, und sie verbindet das Ergebnis der Ausdrucksrelation (= die Aussage) mit dem Ergebnis der Auffassungsrelation (= dem Sachverhalt).

Wo stehen wir jetzt mit dem Bedeutungsproblem? Nun, aus dem naiven Referenten als dem Realbezug und Wirklichkeitsgarant des Sprechens ist etwas ganz anderes geworden: eine Größe nämlich, die nur in ihrer hoch variablen Beziehung zum Aussageinhalt überhaupt in die Bedeutungsrelation eingeht. Die Referenz im herkömmlichen Sinne von Ogden \& Richards ist in diesem Modell so etwas wie ein Grenzfall der letzten Erdung von Aussageinhalten in der jeweils fallweisen Aussagegrundlage. Das ganze Modell ist vom Satz, vom Satzinhalt, von der Proposition (modern gesprochen) her gedacht - und eben nicht vom naiven Realkontakt der Referenz auf außersprachliche Objekte.

Was Gomperz (1908) als Aussagegrundlage fasst, das begegnet uns vorher ähnlich etwa in Philipp Wegeners (1885) „Situation der Anschauung“ und nachher in Gardiners (1932) Modellanekdote von dem Paar, das spazieren gehen möchte und den Regen draußen wahrnimmt. Aussageinhalt und Aussagegrundlage gehen in die Bedeutungsrelation (= Relation zwischen zwei Relationen) nur gemeinsam ein. Es begegnet uns sinngemäß auch (als „,context of situation“) in Malinowskis Anhang zu Ogden \& Richards (1923). Bei Wegener, Malinowski und Gardiner freilich ist der Zusammenhang nicht epistemisch von der Darstellung her gedacht, sondern pragmatisch-kommunikativ von der Situation her. Das epistemische Wahrheitsproblem konturiert sich nur im Verhältnis von Aussageinhalt und Aussagegrundlage: ,der Inhalt des Satzes ,Dieser Vogel fliegt ${ }^{6}$ ist in gleicher Weise, ob nun dieser Vogel wirklich fliege oder nicht", schreibt Gomperz (1908: 67). Das Wahrheitsproblem gehört nicht zur semantischen Relation, denn die ist als Relation zwischen Aussage und ausgesagtem Sachverhalt eine Beziehung zwischen zwei durchweg sprachlichen Größen. Eine davon ist eben die noetische:

Die Bedeutungsbeziehung zwischen Aussage und ausgesagtem Sachverhalt ist also eine Beziehung zwischen zwei teilweise koinzidierenden Gliedern und deshalb keine äußerlich-konventionelle, sondern eine innerlich-begründete Beziehung. (ibid:: 70)

Was Gomperz als Aussagegrundlage bezeichnet (und sowohl gegen den noëtischen Aussageinhalt als auch gegen den semantischen Sachverhalt absetzt), das findet ein Echo nicht allein in dem berühmten Paragraphen 4.024 aus Wittgensteins Tractatus (1971 [1922]: 36): „Einen Satz verstehen, heißt, wissen 
was der Fall ist, wenn er wahr ist. (Man kann ihn also verstehen, ohne zu wissen, ob er wahr ist)“. Auch später, in den Notizen der „Philosophischen Grammatik“, kommt Wittgenstein auf diesen Komplex zurück mit der Frage:

Was es macht, dass man meint, der Gedanke, der gedachte Satz, enthalte die Realität, ist, dass man bereit ist von ihm zur Realität überzugehen und diesen Übergang als etwas bereits in ihm potentiell enthaltenes empfindet. [...] Wir empfinden diesen Übergang so legitim, wie den erlaubten Zug eines Spiels. (Wittgenstein 1973: 154)

Was aber nicht davon ablenken sollte, dass wir es in unserer alltäglichen Kommunikationsrealität nicht sehr häufig mit Sätzen zu tun haben, deren Wahrheit empirisch an einer aktuellen und geteilten Situation überprüft werden kann oder muss. Die geteilte Situation dient eher der Fundierung von Relevanz als der Etablierung von Wahrheit. Wir begnügen uns in der Regel mit dem, was Gomperz als selektive „Auffassung“ der Situation oder Aussagegrundlage einführt (Gomperz 1908: 61, 77). Die Aussagegrundlage als solche ist für Gomperz lediglich das noch nicht aufgefasste Korrelat unserer sprachlichen Aktivität. Allein diese letztere erlaubt Rückschlüsse darauf, wie die aktuellen Gegebenheiten von Teilnehmern aufgefasst werden. Die Beziehung zwischen Aussagelauten und Aussagegrundlage bleibt bei Gomperz unter dem Namen „Bezeichnung" eher „lose und äußerlich“ (ibid.: 64).

In der späteren Revision seiner Bedeutungslehre (Gomperz 1953 [1941]: 251274) orientiert sich Gomperz an der Semiotik von Charles Morris und am Programm der Klärung von fachlichen und alltäglichen Ausdrucksbedeutungen, mit vorsichtiger Kritik am Logischen Positivismus und mit der bescheidenen Erwartung, dass die Klärung der Bedeutungen womöglich nicht weit über das hinausführt, was wir ohnehin bereits wissen. Fest hält er jedoch an der Erkenntnis von 1908, dass es falsch ist zu sagen, Namen würden Objekte bedeuten und Sätze Tatsachen beschreiben (vgl. ibid.: 261). Jede Situation kann durch zahllose „statements" aufgefasst werden. Wie weit sich gleichwohl der späte Gomperz von seinen sehr „deutschen“ philosophischen Grundlagen entfernt (und dem USamerikanischen Pragmatismus angenähert) hat, erhellt aus der (autobiographischen) Bemerkung, am Ende zählten nicht die metaphysischen Systeme selbst, sondern der ,practical outlook“, der ihnen unterliege oder unterlegt werde (ibid: 22).

Gomperz' frühe Konstruktion eines Modells für Semantik und Noetik kommt darin mit Husserl überein, dass beide den Nullpunkt der Betrachtung verschieben, hin zum Sprecher, der in der Verwendung sprachlicher Ausdrücke deren noetischkonstruktives Potential nutzt.

Interessant ist aber auch, wo Gomperz (1908) sich von Husserl ausdrücklich abgrenzt. Gegen dessen Akttheorie der Bedeutung macht er (ibid:: 217) geltend, sie durchschneide das Band zwischen Aussageinhalt und Aussagegrundlage. Das kann man interpretieren als eine Art Ehrenrettung der empirischen Kommunikationssituation gegen den absolut monologischen Idealismus des frühen Husserl, den Bühler (1934: 66) als „Diogenes im Fass“ karikiert, weil er zunächst nur wenig Sinn dafür hat, dass sprachliche Bedeutungen und ihre intentionalen 
Gegenstände interaktiv geteilt sein müssen (in einem dringend zu spezifizierenden Sinne), wenn sie für das Denken und für die Verständigung einen Nutzen haben sollen. Maas (2016: 351) weist allerdings darauf hin, dass Husserl in seinem Spätwerk den Schwenk vom einsamen Seelenleben hin zur „Bindung der Zeichenstruktur an Geltung für andere" ebenfalls vollzogen habe. Ich komme darauf zurück.

In der Tat nähert Gomperz (1908) sich mit seinem Blick auf die Aussagegrundlage, die geteilte außersprachliche Szene von Sprecher und Hörer, wieder den Bedürfnissen einer realistischen „Linguistik des Sprechens“ - auch wenn Husserl durchaus philosophisch strenger und konsequenter denkt (und Gomperz' „pathempirischer“ erkenntnistheoretischer Unterbau für semantische Zwecke völlig überflüssig ist). Während Husserl die Umfelder und Gegebenheiten des jeweiligen Sprechens, in denen die fallweise „Bedeutungserfüllung“ stattfindet, phänomenologisch „einklammert“, baut Gomperz (ibid.) darauf, dass es zwischen Aussageinhalt und Aussagegrundlage so etwas wie ein Relevanzverhältnis gibt (er ist gewissermaßen ein Ahne von Sperber \& Wilson 1986!). Husserl ist da (sagen wir) passiver, er konzediert die Möglichkeit der Fundierung noetischer Kategorien in der Anschauung, diese Fundierung sei aber keinesfalls identisch mit dem Intendierten (Husserl 1913 [1901]: 132 ff.):

Nicht im wechselnden Inhalte, sondern in der Einheit der auf die konstanten Merkmale gerichteten Intention liegt das Wesentliche der Sache. (ibid: 136)

Bedeutung, so ist Gomperz' Definition zu resümieren (Gomperz 1908: 68-70), ist die Relation zwischen den beiden selbst relationalen Größen Aussageinhalt und Sachverhalt, zwischen zwei überwiegend sprachlichen Ebenen, deren eine aber mit der (außersprachlichen) Aussagegrundlage verzahnt, in dieser geerdet ist. Keineswegs gelten ihm Aussagen und Ausdrücke als bedeutungsgleich, die sich auf die gleiche Aussagegrundlage beziehen (wie es dann bei Ogden \& Richards 1923 praktiziert wird), und jeder Aussagegrundlage ist es vollkommen äußerlich, wie sie von Sprechern sprachlich gegliedert und akzentuiert wird. Wie pragmatiknah Gomperz (ibid.: 70) hier bereits denkt, erhellt aus seinem Beispiel: Der Sprecher gliedert die Aussagegrundlage so, dass eine lokal relevante Bearbeitung derselben geschieht. Mit der Aussagegrundlage der Äußerung: $D a$ ist eine Schlange! wären unendlich viele Aussageinhalte kompatibel: Da hat sich was bewegt! Ein Tier! etc., aber der Hinweis auf die Schlange führt zu „zweckmäßigem" Hörerverhalten. Auch ob eine gegebene Aussagegrundlage propositional oder bloß nominativ adressiert wird (Da kriecht eine Schlange vs. eine Schlange) liegt ganz beim Sprecher und nicht in der Situation. Immer wieder macht Gomperz klar, wie different, ja gegensätzlich Aussageinhalt und Aussagegrundlage sind:

Man stellt sich dann vor, es könnten eben nur jene Aussagegrundlagen durch einen bestimmten Aussageinhalt aufgefasst werden, welche diesen schon in sich enthalten. (ibid: : 145)

So spottet er. Der Aussageinhalt sei aber eben eine außerempirische Wesenheit, die nicht in den empirischen Elementen der Aussagegrundlage enthalten sein 
könne. Anschauungen sind durch Sprache nicht mitteilbar, wird Marty (1925: 87) in den erst post mortem veröffentlichten Fortsetzungen zu seinem Hauptwerk (Marty 1908) schreiben. Der Aussageinhalt hingegen kann seinerseits nicht wahrgenommen oder vorgestellt werden. Seine „Gegenständlichkeit“ ist noetisch, er ist ein „Gegenstand höherer Ordnung“" (Gomperz 1908: 91 bzw. 100 f.) ${ }^{21}$.

„Verstandesgegenständlichkeit“, so wird Husserl später (1948 [1938]: 282285) schreiben, beruht auf prädikativen Leistungen, und die sind gerade nicht fundiert in der vorprädikativen und passiven Rezeptivität. Und weiterhin beruht „Verstandesgegenständlichkeit“ auf der Reflexivität der Zuwendung zu prädikativen Leistungen. Porzig (1950) bezieht sich in Fragen der Abstraktion ausdrücklich auf Husserl (auch schon in dem 20 Jahre vorher erschienen klassischen Text über „Die Leistungen der Abstrakta in der Sprache“).

Gegen den psychologischen Individualismus der Ausdruckslehren seiner Zeit (und implizit auch gegen Husserl) bemerkt Gomperz (1908: 237), das Auszudrückende möge ja so persönlich sein wie es wolle, der Sprecher habe immer nur die überlieferten Formen, Bedeutungen und Schemata, die sich seiner Spontaneität weitgehend entzögen. Die Traditionen des Sprechens begrenzen, kanalisieren, konventionalisieren den individuellen Ausdruck. In diesem Punkt ist es einmal Husserl, der eher hörerbezogen argumentiert und darauf verweist, dass per Kundgabe der Hörer eine (freilich indirekte, vermittelte) äußere Wahrnehmung der inneren Erlebnisse des Sprechers hat (Husserl 1913 [1901]: 34). Es ist wiederum Bühler (1927, 1933), der diesen Gegensatz ausdruckstheoretisch fruchbar macht (vgl. Friedrich 2010).

Was Husserl phänomenologisch „einklammert“: die fallweisen „bedeutungserfüllenden Akte“ im Unterschied zu den „bedeutungsverleihenden Akten“, das entspricht in der Sache der variablen „Bündigkeit“ sprachlicher Ausdrücke in den wechselnden Umfeldern des Sprechens bei Bühler (1934). Und es entspricht theoriearchitektonisch Gomperz' Aussagegrundlage. Verständigungstechnisch gilt: Die Relevanz der noetischen Bezüge erweist sich, indem sie in der Aussagegrundlage verankert werden können. Erst in der Zuwendung lösen sich Gegenstände aus dem Sinnesfeld, wird Husserl (1948 [1938]: 75) später sinngemäß über das elementare Wahrnehmungsurteil schreiben.

In der Bedeutung eines Ausdrucks (so Husserl 1913 [1901]: 54) konstituiert sich die Beziehung auf den Gegenstand, egal, ob der Gegenstand existiert, fingiert oder auch unmöglich ist wie rundes Viereck oder goldener Berg. Referentiell betrachtet ist ein Ausdruck bedeutungslos, wenn es keinen Gegenstand gibt, auf den er sich beziehen ließe. Ogden \& Richards (1923) werden aus diesen Überlegungen das Wortmagie-Verdikt gegen alles machen, was sich nicht in Zeichen-Referent-Komplexe pressen lässt.

21 Die Rede von den „Gegenständen höherer Ordnung“ taucht bei Bühler (1934: 57 ff.) im Zusammenhang mit den „Sprachgebilden“ wieder auf, akzentuiert auf die kategorialen Konstruktionsgefüge der Wortarten und Satzglieder. Als versierter Philosophiehistoriker lässt Gomperz (1908) den Leser auch nicht im Unklaren darüber, dass sein „Aussageinhalt“ nach dem Modell des „Lekton“ der Stoa gebaut ist, das seinen Urhebern ebenfalls weder als subjektiver Gedanke noch als materielle Realität galt, sondern als etwas, was beide Seiten ,objektiv“ verbindet (Gomperz 1908: 80). 
Theorietechnisch hat Gomperz (1908) einen Vorteil gegenüber Husserls Logischen Untersuchungen, indem er auf der eigentümlichen Rolle der „Aussagegrundlage" für das Bedeutungsproblem besteht und den Monologismus und Internalismus Husserls kritisiert. Gomperz (ibid.) hat ein Modell dafür, dass die sprachlich gegliederte Proposition (modern gesprochen) ebensowohl für sich stehen (und für sich interpretiert werden) $\mathrm{kann}^{22}$, als auch genommen werden kann für eine Instruktion, welche die aktuelle Aufmerksamkeit der Teilnehmer in einer gegebenen Interaktionssituation auf deren für relevant erachtete Aspekte koordiniert. Damit nähert er sich einer Erkenntnis, die in der neueren Psycholinguistik des Sprach- und Redeverstehens nach zahllosen Ab- und Umwegen erneuert wird: der Erkenntnis nämlich, dass „Referenz“ als mapping und Aufmerksamkeitssteuerung im geteilten perzeptiv-aktionalen Orientierungsfeld gelernt wird, bevor noetisch generalisiert auf alles Bezug genommen werden kann, was im aktuellen und lokalen Orientierungsraum des laufenden Diskurses konstruiert und indexikalisch adressiert werden kann.

In Erfahrung und Urteil kommt Husserl (1948 [1938]: 285 ff.) dann noch einmal ausdrücklich auf das Gomperz-Problem zu sprechen - freilich ohne diesen ausdrücklich zu nennen. Als „Sachlage“ fasst er das, was Gomperz „Aussagegrundlage" nennt. Dabei handele es sich um passiv konstituierte Verhältnisse, über die Urteile in ganz verschiedene Richtungen gebildet werden können. Ganz wie Gomperz, aber eben in eigener Terminologie, versteht er die „Sachlage“ als passives Fundament für „Sachverhalte“ im eigentlichen Sinne (ibid.: 288-291), also für das, was wir heute eher als Propositionen bezeichnen würden ${ }^{23}$.

Somit ist „Referenz“ bei Licht besehen ein quasi-terminologisches Kürzel für sehr heterogene Zielgrößen: Referenz ,endet“ oder „mündet“ nie im außersprachlichen Gegenstand, sondern in der auf diesen gerichteten Aufmerksamkeit. Niemals ist sie das, was Ogden \& Richards (1923) aus ihr machen: ein außersprachlicher Garant für die Wahrheits- und Erkenntnisfähigkeit des Sprechens.

In der Welt des psycholinguistischen Laborexperimentes nehmen Studien zum Referenzproblem fast automatisch die (objektivierbare und reproduzierbare) Form der sprachlichen Instruktion für die Auswahl eines von mehreren möglichen Referenzgegenstandes an. Das gilt für die deutsche Tradition der „,referentiellen Kommunikation" (vgl. das Referat bei Mangold-Allwinn et al. 1995) ebenso wie für die angelsächsische (etwa Clark \& Wilkes-Gibbs 1986). Ein jüngst erneuerter psycholinguistischer Befund (Garrod 2011) besagt, dass wir sprachlichen Input da, wo relevante perzeptive und aktionale Bezüge zur Verfügung stehen, opportunistisch auf diese abzubilden versuchen (vom Koaktionsfeld über den Film bis zum Comic!) - was etwa bei einem Leseexperiment mit schriftsprach-

22 In der Terminologie von Gomperz (1908) ist sie dann ein rein noëtisches Gebilde.

23 Und auch Bühlers „Sachverhalte“ sind, wie gesagt, in diesem Sinne noetische Gebilde, keine ontologischen. Wo Bühler selbst die Punkte referiert, an denen er über Gomperz (1908) hinausgeht, da nennt er zweierlei: das (an der Phonologie entwickelte) Prinzip der abstraktiven Relevanz, das auch für die sprachliche Darstellung gelte, sowie den „Verweis auf das Faktum des Sprechverkehrs, welcher, wie ich glaube, ohne das Eingehen auf erlebnispsychologische oder ontologische Fragen eine Entscheidung gestattet" (Bühler 1934: 225). Im Sprechverkehr ist es die Aufmerksamkeit, die indexikalisch koordiniert wird mit Bezug auf Sprechsituation oder Aussagegrundlage. 
lichem Text natürlich gar keinen Sinn ergibt, weil dieser uns unmissverständlich auf den Bezugsraum des lokalen Diskursmodells verweist, in dem Bezüge dann allein bündig werden können. Die sprachliche Richtung und Koordination von Aufmerksamkeit ist die Klammer, die beide Prozesse, sprachliche Orientierung und Auswahl eines Bezugs, miteinander verbindet. Die (von Husserl „eingeklammerte") fallweise wechselnde Bündigkeit sprachlicher Ausdrücke (Erfüllung in der Anschauung etc.) macht aber insofern einen Unterschied, als es ohne intersubjektive Erdung des Sprechens in der „Aussagegrundlage“ keine Basis für die Objektivitätssuggestion noetischer Objekte gibt ${ }^{24}$. Auch mit Bezug auf die biologische Sprachevolution dürfte es keinen Zweifel daran geben, dass wir zunächst darauf programmiert sind, sprachliche Kommunikationen auf die aktuell geteilte Wahrnehmungswelt zu beziehen und irgendwie in dieser zu verankern:

The language learner is innately predisposed to assume that communicative acts refer to the world. Thus, from the outset, the language learner attempts to compute the referential implications of the linguistic characterizations of the input. (Trueswell et al. 2011: 71)

Dieses psycholinguistische Zitat aus der jüngeren Gegenwart ist auch darin der schlichten Referenzideologie überlegen, dass von den Aufmerksamkeitsimplikationen der Äußerung die Rede ist, nicht von singulären Gegenständen, die repräsentiert würden. Die vermeintlich einfach „gegebenen“ Objekte sind Projektionen unseres (sensomotorischen und symbolischen) Umgangs mit ihnen, die intentionale Ausrichtung auf sie beginnt bei uns.

Der Eigenname, nicht zufällig referenzsemantisches Paradestück, löst die Suggestion des objektiven Individuenbezugs vollständig von der Anwesenheit des Bezeichneten und zugleich weitestgehend auch von sprachlichem Wissen: Was wir über die Träger von Eigennamen wissen, das ist (abgesehen von Minimalkonzeptualisierungen wie etwa $\mathrm{m} / \mathrm{w}$ bei Vornamen) Sach- und Welt- und kein Sprachwissen. Eigennamen sind gleichsam die perfekten Referenzialisierer, sie identifizieren aperspektivisch, aus allen Lebenslagen den gleichen Bezug, und das ohne Dazwischenkunft „störender“ einzelsprachlicher Konzepte. Es hat keinen Sinn zu fragen, ob „Gretel“ in einem Märchen „,dasselbe“ Gretel ist wie in einem anderen Märchen, schreibt Husserl (1948 [1938]: 202), weil es Individualität, Identität und Identifizierung nur in der Welt der wirklichen Erfahrung gibt.

Die Pointe der universellen, aber eben einzelsprachlich realisierten Noetik liegt hingegen an dem den Eigennamen entgegengesetzten darstellungstechnischen Pol, am Pol der Kombinatorik. Und von diesem Pol aus betrachtet ist Monosemie und Monoreferentialität der Grenzfall, nach dem wir unsere spontane Sprachideologie (linguistic ideology nach Silverstein 1979) modellieren ${ }^{25}$. Das

24 Aus psycholinguistischer Sicht notiert Garrod (2011: 276) über die Differenz zwischen noetischsymbolfeldbasierten Diskursmodellen und perzeptiv-anschaulichen: „In the first case, these will be processes aimed at constructing a coherent and parsimonious model on the basis of what has been read so far. In the second case, they will be processes aimed at establishing mappings between the utterances under interpretation and a model based on the visual world in view. "Dieses letztere dürfte freilich in der Regel auch durch koaktionale Relevanzen strukturiert sein.

25 Silverstein (1979: 193) definiert als linguistic ideology „, any set of beliefs about language articulated by the users as a rationalization or justification of perceived language structure and use“. 
noetische Potential konzeptualisierender und grammatischer Sprachzeichen entfaltet und differenziert sich in den Konstruktionen, in denen die Zeichen vorkommen. Durch lexikalisch basierte Argumentbeziehungen, Kollokationen, Nennbefestigung komplexer Ausdrücke, Typisierung wird der Suchraum für noetische Bezüge ganz ähnlich reduziert wie durch die im Wahrnehmungs- und Orientierungsraum verfügbaren Beziehbarkeiten. Die Nahtstellen des Sprechens zur unmittelbaren Interaktionssituation werden dadurch gelockert. Aber kognitiv ist es in beiden Fällen das Gesprochene selbst, das die Aufmerksamkeit im Suchraum strukturiert und zentralisiert. Aber das ist ein anderes, ein psycholinguistisches Thema. Zurück zur semantischen Theoriegeschichte.

\section{ANKNÜPFUNGEN UND WEITERUNGEN: JENSEITS DER REFERENZ}

Bühlers (1934: 62 ff.) - durchaus anerkennende - Kritik an Husserls subjektiver Aktlehre trifft den Kern des Noetikproblems: Was Husserl als „Komplexionsgesetze" einer reinen Grammatik postuliert, das - so Bühler (ibid.: 65) - funktioniert nicht auf der Grundlage grammatischer Konstruktionsmodelle allein. In Ihren Gefügen (heute würde man sagen: Konstruktionen) bauen natürliche Sprachen ergänzend immer auch auf das „Sachwissen der Empfänger“ (ibid.: 65). Sachgesteuerte Selektionen des Hörers seien an jedem Bedeutungsaufbau mit beteiligt. Und hier kommt Bühler (freilich ohne den Namen zu nennen) auf Bréals (1868) Kerngedanken zurück:

Die sprachliche Darstellung lässt allenthalben Spielräume der Bedeutungsunbestimmtheit offen, die auf keine andere Weise wie durch den Hinblick auf die „objektiven Möglichkeiten“ geschlossen werden können und in jeder menschlichen Rede auch faktisch geschlossen werden. Wäre dem nicht so, dann hätten es die Lexikographen leichter; das ist wahr. Aber die natürliche Sprache wäre um das Erstaunlichste und praktisch Wertvollste, was ihr eignet, verarmt. Verarmt um die erstaunliche Anpassungsfähigkeit an den unerschöpflichen Reichtum des im konkreten Falle sprachlich zu Fassenden; und dies macht, von der anderen Seite gesehen, die Freiheitsgrade der Bedeutungsverteilung möglich und damit die Husserlsche Aktlehre allererst notwendig und unentbehrlich auch für eine im ersten Anlauf „objektive“ Sprachanalyse. (Bühler 1934: 66)

Es sind die Konturen einer noetischen Theorie der bedeutungsverleihenden Akte, die (hierin durchaus Gomperz verwandt) in der Sozialsphäre des geteilten Sachwissens abgestützt ist, ohne doch ihre Bedeutungen einfach in den bezeichneten Sachen bündig werden zu lassen. Auf Schritt und Tritt baut die Sprechpraxis auf „entgegenkommendes“ Hörerwissen und offenbart damit ihren indexikalischen Charakter.

Auch bei Roman Jakobson, der ja stark von Bühler geprägt ist, finden wir eine ähnliche Rezeption und Weiterentwicklung von Husserls Aktlehre, hin zu Intersubjektivität, Perspektivität und Aktivität der Bedeutungsproduktion (vgl. Holenstein 1975: 70). Zu den genuin sprachlichen Ressourcen des Nennens fügt Jakobson (auch das im Geiste Bühlers) noch den Argumentstatus des Genannten im Blick auf das Prädikat, so dass eine doppelte Architektonik entsteht: Auf der 
Ebene der Bedeutungskomplexion kann das Genannte als Substrat von Attributen (bottom-up) und als Argument eines Prädikats (top-down) gedeutet werden. Vom schlichten Referenzialismus Freges distanziert sich Jakobson später ebenfalls: Das klassische Morgenstern - Abendstern - Beispiel (bei Frege referenzgleich, aber bedeutungsverschieden - freilich in Freges eigener Sinn/BedeutungTerminologie) beurteilt er (vgl. ibid.: 95) später auch als referenzungleich, weil auf unterschiedliche Phasen des Bezugsgegenstandes abgestellt.

Eine angelsächsisch pragmatisierte und gut durchdachte Version des noetischen Grundgedankens liefert schließlich Alan Gardiner ${ }^{26}$ in seiner (zuerst 1932 und 1951 in zweiter Auflage erschienen) Theory of Speech and Language. Husserl scheint er in erster Linie über den Appendix von Ogden \& Richards (1923) zu kennen, deren Epistemologismus er aber keineswegs teilt. Mehr abgewinnen kann er Malinowskis (als Anhang bei Ogden \& Richards 1923 reproduzierter) Lehre von der phatic communion, der Kontakt- und Gemeinschaftsfunktion des Sprechens. Gewidmet ist das Buch Wegener (1885), dem der Autor sich sehr verbunden fühlt.

Gardiners Formel für den Status des thing-meant lautet: außersprachlich, aber nicht ontologisch, ,accepted merely for the purposes of speech" (Gardiner 1951: 31), abgestellt (wie Gomperz) auf Realkontakt, aber eben nicht auf Referenz im herkömmlichen Sinne: „This in-and-out-of-reality is characteristic of all speech“, schreibt er (ibid.: 32). Ohne sprachlichen Zugriff können wir zumal abstrakte Gegenstände nicht auffassen (nomina si nescis, perit et cognitio rerum).

Dass sprachlich alles in nennbare Gegenstände verwandelt werden kann, auch ganze Propositionen, ist ihm vertraut (ibid.: 23-27). Er erneuert den modistischen Gedanken, alle Wörter seien Namen von „Dingen“, unterschieden sich aber nach ihrer „mode of presentation“. Am Beispiel Religion argumentiert er, dass auch Abstrakta ein thing-meant haben, das sich von der Bedeutung, dem meaning des Ausdrucks, abhebt: Nicht die Bedeutung des Wortes Religion sorge für Aufregung, sondern das Gemeinte (ibid.: 30). Für Logiker seien alle wasbestimmten Ausdrücke so etwas wie ein Prädikat, und ihr thing-meant sei das Subjekt dazu. Bei allen Ausdrücken ist das thing-meant außerhalb des Wortes - ein durchaus Husserlsches Prinzip. Alle Wörter seien typisierende „class names“, schreibt er (ibid.: 38), und entwickelt ganz beiläufig den Grundgedanken der Prototypensemantik, sowie, diese ergänzend, eine Theorie der „selective attention", der selektiven Aufmerksamkeit, die lokal für das thing-meant irrelevante Aspekte der Bedeutung ausblendet (ibid.: 48). Aus dem instrumentellen Charakter sprachsystemischer Bedeutungen folgert Gardiner (ibid.: 100), dass meaning alleine in den bedeutungsverleihenden Akten der Sprecher residiere, nicht in den Zeichen und Symbolen an sich. Die Verwendung des Ausdrucks meaning auch für das extrakommunikativ betrachtet Zeichen verwirre die Verhältnisse zwischen meaning und thing-meant - was man im Blick darauf, dass Wörterbücher bis 
heute bisweilen extrem bezeichnungsorientiert vorgehen, durchaus bestätigen kann.

Interessant ist Gardiner besonders da, wo er über die monologischen Perspektiven des frühen Husserl deutlich hinausgreift: Differenzen zwischen Sprecher- und Hörerbedeutung sind durchaus real, werden aber dynamisch minimiert, insofern der Sprecher immer auch Hörer (seiner selbst wie der anderen) ist und daher alle Wörter mit ihren Bedeutungen aus beiden Perspektiven kennt und sie auszugleichen vermag (ibid.: 112).

Metaphern sind für Gardiner eine Art durchlässiger Austauschmembrane zwischen meaning und thing-meant, zwischen language and speech. Wenn sie deckend (kongruent in der Terminologie von Wegener 1885) geworden sind, importieren sie Merkmale ihrer things-meant in die Bedeutungssphäre der Einzelsprache.

In Gardiners origineller Lehre gehören die Wortarten und Wortformen mit ihrem kombinatorischen Potential zur Sprache, die Sätze und ihre Konstituenten hingegen rechnet er (hierin Saussure ähnlich) zur Rede (speech). Er moniert die Bezeichnung parts of speech für die Wortarten (Gardiner 1951: 134). Über die Wortart Adjektiv schreibt er:

The adjective is a word congenitally so constituted as to exhibit its meaning and to hide its thing-meant. (ibid.: 260)

Was im Großen und Ganzen der modernen Auffassung von der modifizierenden kategorialen Bedeutung der Adjektive (und Adverbien) entspricht, die koprädikativ, koreferentiell, konzeptmodifizierend etc. spezifiziert sein kann durch die syntaktische und sonstige Umgebung des Wortes und durch die Umfelder des Sprechens. Modifizieren ist die noetische Grundlage der Adjektive in natürlichen Sprachen, Nennen und Identifizieren die der Substantive.

Einige knappe Bemerkungen noch zu Erwin Koschmieder (1965) ${ }^{27}$. Bei ihm, dem heute weitgehend unbekannten Slawisten, verbinden sich die Motive, deren Stunde dann im Internalismus, Universalismus und Formalismus der generativen Syntax gekommen ist. Koschmieder formuliert ein noetisches Programm für die Syntax mit den drei Dimensionen Nennung, Verzeitlichung und Leistung. Diese drei Dimensionen werden in jeder Einzelsprache anders organisiert. Am Übersetzungsproblem ${ }^{28}$ sucht er zu zeigen, dass das Gemeinte (in gewissen Grenzen) universal ist. Die Vermittlung zwischen einzelsprachlicher Grammatik und universellen noetischen Zielgrößen geschieht via Syntax. Die Syntax bildet das Scharnier (heute würde man wohl interface sagen) zwischen den einzelsprachlichen Formen und Konstruktionen und den universalen Kategorien des Gemeinten. Die Kalkülisierung und Formalisierung der Syntax ist ausdrücklich Programm bei Koschmieder (ibid.: 85). Es ist die Kombinatorik, mittels derer das einzelsprachliche Zeicheninventar ausgebaut und entwickelt wird. Die Syntax

27 Die einschlägigen Aufsätze Koschmieders aus den 1940er und 1950er Jahren sind zugänglich in Koschmieder (1965). Koschmieders Verbindung zu Husserl ist stärker über den Husserl-Schüler Ludwig Landgrebe vermittelt (Husserl 1948 [1938]).

28 „Das Verfahren der Sprachwissenschaft hat auch bisher stets diese Übersetzbarkeit zur Voraussetzung gehabt“" (Koschmieder 1965: 135). 
wird zur noetischen Schlüsseldisziplin, aber eben nicht als Aufzählung ihrer einzelsprachlichen Besonderheiten, sondern in ihrer Beziehung auf die Zielgrößen, die allen Sprechern natürlicher Sprachen gemeinsam sind. Koschmieders Version des Zeichendreiecks besteht aus den (lateinisch bezeichneten) Größen: signum, designatum und intentum. Ein Referent kommt nicht vor. Seine Rolle übernimmt das Intendierte, das Gemeinte.

Historiographisch interessant ist die Tatsache, dass Koschmieder mit den zu seiner Zeit in der akademischen Sprachwissenschaft sehr mächtigen Vertretern des einzelsprachlich-volkhaften Weltbildes, mit Leo Weisgerber und seiner Sprachinhaltsforschung, theoretisch frontal zusammenstoßen musste mit seiner universalen Noetik. Seine Gewährsleute findet er eher in Skandinavien (Otto Jespersen, Louis Hjelmslev, Viggo Brøndal). In einem Beitrag, der pikanterweise Leo Weisgerber zu dessen 60. Geburtstag gewidmet ist, hat Koschmieder (vgl. ibid.: 176-188) dessen einzelsprachliches Weltbild höflich, aber bestimmt widerlegt: Gemeint wird durch die einzelsprachlichen Mittel, aber eben nicht deren einzelsprachliche Inhalte.

Mit Gardiners thing-meant verbindet ihn der Sinn dafür, dass es in der Noetik eine pragmatische Dimension gibt (Koschmieder nennt sie die noetische Dimension der Leistung). An Beispielen, die deutlich Austins Sprechakttheorie vorwegnehmen, zeigt Koschmieder (ibid:: 88), wie noetische Festlegungen teils in den Umständen des Sprechens liegen, teils explizit versprachlicht werden. Der deutsche Satz:

Ich bitte (hiermit/gerade) um Verlängerung der Leihfrist meiner Bücher.

Steht in seiner noetischen Leistung einmal für die Dimension von Bericht/Darstellung, etwa als Antwort auf die Frage eines Bekannten:

Was machst du denn hier?

Oder aber für den performativen Akt (modern gesprochen), wenn gegenüber dem Bibliotheksmitarbeiter geäußert. Die Ausdrücke hiermit und gerade versprachlichen die noetische Differenz eindeutig, entweder im Sinne der performativen Lesart (hiermit) oder im Sinne der verzeitlichenden Lesart (gerade).

Das Panorama dieser „alternativen“ Semantiken ließe sich erweitern, nähme man die kultur- und kommunikationssoziologische Rezeption des fraglichen Diskurses hinzu, die (wie eingangs erwähnt) nur teilweise in Deutschland (prominentes Beispiel ist Freyer 1923), zum anderen Teil aber in den USA stattfindet (z. B. Znaniecki 1919; Sapir 1927, 1931). Bei Sapir $(1927,1931)$ ist es der Grad des kulturellen Vorverständigtseins, der die Ökonomie der erforderlichen symbolischen Mittel reguliert, und diese letzteren ,have the property of locating every social referent" (Sapir 1931: 78). Die Teilnehmerperspektive mit all ihren praktischen Selbstverständlichkeiten macht blind für die explizite Erkenntnis kulturspezifischen Regeln und Formen, die jedoch keine scharfen Grenzen des Meinbaren definieren. Die Dingwelt wird nicht als solche aufgefasst, sondern nach ihrer Relevanz für geteilte Handlungsmuster. All das wird bei Sapir vorgetragen und entwickelt in nüchtern pragmatischer Diktion und ganz ohne den konnotativen Überschwang der deutschen geisteswissenschaftlichen Terminolo- 
gie, die bei Freyer (1923) dominiert und seine Texte heute so schwer verdaulich macht (,objektiver Geist“ etc.). In den USA führt diese Linie zur Ethnolinguistik und schließlich zur ethnomethodologischen Soziologie. Aber das ist eine andere Geschichte.

Nur eine Bemerkung zu Freyers (ibid.) Einleitung in die Kultursoziologie sei noch erlaubt, da dieser eine Lesart der intentionalen Semantik etabliert, die weit über das Sprachliche hinausführt und neben den Zeichen auch „Gebilde“, „Geräte“ und „Sozialformen“ in den Raum des kommunikativ Intendierten einführt (vgl. ibid.: 55-74) - und insofern bereits an moderne Akteur-NetzwerkTheorien erinnert. Für Freyer ist bereits die hinweisende Gebärde (konventionell bloß Hinweis auf einen in der koordinierten Aufmerksamkeit gegebenen Gegenstand) auf dem Weg zur intentionalen Fixierung von Sachverhalten:

Ein anderer weist mich, indem er den Zeigefinger ausstreckt, auf das Buch hin, das ich suche. Was ist der schlichte Sinn dieses Zeichens? Offenbar der Satz: „Dort liegt das Buch." Dieses Zeichen sagt nicht das geringste über die seelische Lage desjenigen, der es gibt. [...] Deren [der Geste; CK] schlichter Sinn, wie gesagt, ist der Satz: „Dort liegt das Buch“ und nichts sonst. Natürlich nicht in der sprachlichen Formulierung, nicht als Satz, streng genommen überhaupt nicht als Urteil. Der Sinn jener Gebärde ist nicht sowohl ein explizites Urteil als der Sachverhalt, „dass dort das Buch liege“; jenes Objektiv also, das den Inhalt auch des Urteils bildet. Urteil und Gebärde haben diesen Sachverhalt zum gemeinsamen Gegenstand ihrer Intention. (ibid:: 20 f.)

Die hinweisende Geste steht insofern für die erste Stufe der kulturellen Objektivation, als von ihrer situierten Leistung nichts zurückbleibt, was zur Weiterverwendung taugt. Die Geste geht in ihrer fallweisen Leistung auf. Anders verhält es sich bereits bei sprachlichen Ausdrücken, die neu arrangiert und in wechselnde Umfelder eingesetzt werden können, erst recht bei Geräten und Sozialformen ${ }^{29}$.

\section{SCHLUSS}

Was hier selektiv und in groben Umrissen verdeutlicht werden sollte, das summiert sich zu den Anfängen einer nicht-referentiellen Semantik des intentional Adressierbaren, des Gemeinten. Im Problemhorizont der gegenwärtigen linguistischen Semantik wirken diese Bestrebungen unzeitgemäß. Sie entsprechen nicht dem stärker szientifizierten Denkstil der vorherrschenden referentiellen Semantik.

Das war um 1900 herum offenbar anders, wie man an der damaligen Konvergenz ganz gegensätzlicher Bemühungen sehen kann: Michel Bréal, Arsène Darmesteter in Frankreich, Fritz Mauthner in Deutschland (mit der suggestiven Formel, Bedeutung sei nicht im Kopf des einzelnen und auch nicht in der „substantivischen“ Dingwelt, sondern entstehe „Zwischen“ den Menschen), Karl Otto Erdmann, Philipp Wegener und viele andere mehr (vgl. Nerlich 1992). 
Zu den für die linguistische Semantik axiomatisch wesentlichen Erkenntnissen von Gomperz und Husserl (beim letzteren deutlicher herausgearbeitet; vgl. Husserl 1913 [1901]: 12-14 und öfter) gehört die noetisch (sagen wir) generative ${ }^{30}$ Dimension der Syntagmatik und Kombinatorik der Sprachzeichen. Was Bréal (1868) als idées latentes bezeichnet, die Tatsache nämlich, dass die manifeste grammatisch-kategoriale Konstruktion lediglich ein in der Rezeption auszufüllendes System von Hinweisen auf die Bezüge gibt, dass Bedeutung eben darin besteht, die Bezüge auf das Gemeinte zu organisieren (und nicht einen Spiegel oder ein genaues Modell derselben zu geben), das zieht sich auch durch die Arbeiten von Husserl und Gomperz.

Bedeutungsanalyse dürfe sich durch grammatische Analyse nicht gängeln lassen, manchmal seien grammatische Unterschiede wesentlich, manchmal zufällig. Wir hätten aber die natürliche Neigung, hinter jedem grammatischen Unterschied einen logischen zu sehen (wie wahr!). Die Kategorien des Gemeinten spiegeln sich nicht exakt in den einzelsprachlichen Kategorien und Konstruktionen, in denen sie realisiert werden ${ }^{31}$. Und es gibt auch keinen Parallelismus zwischen der "Zusammengesetztheit der Bedeutungen“ (Husserl 1913 [1901]: 296) und der Zusammengesetztheit der noetischen Gegenstände, auf die sie sich beziehen. Das ist bei Husserl und seinen Zeitgenossen sicher gegen Wilhelm Wundt und seine umfassende Parallelitätshypothese für Ausdruck und Ausgedrücktes gerichtet. Es ist aber zentral auch für die späteren Debatten über Kompositionalität in der Semantik. Als Beispiel für eine einfache Bedeutung, die einen komplexen Gegenstand meint, nennt Husserl (ibid.: 298) den Eigennamen Schulze (jedes Individuum ist unendlich zusammengesetzt!), und als Beispiel für eine zusammengesetzte Bedeutung für einen einfachen Gegenstand nennt er (etwas launig) den Ausdruck einfacher Gegenstand, der ja per definitionem eben das meint, obwohl er selbst zusammengesetzt ist. Bei Gomperz ist der antiparallelistische Modellgedanke ähnlich, aber (wie gehabt!) stärker auf Kommunikation und Aufmerksamkeitslenkung beim Hörer bezogen. Über das Auffassen der noetischen Gegenstände heißt es:

Auffassen heißt jetzt: in einer bestimmten Weise, in einem gewissen Lichte betrachten: durch eine gewisse Verteilung der Aufmerksamkeit in bestimmter Weise gliedern. (Gomperz 1908: 184)

Jedes Wort trägt (s)einen Gesichtspunkt an das Gemeinte heran ${ }^{32}$. Bei noetisch geprägten Semantikern wie Hempel (1980) findet man entsprechende Gedanken z. B. in der Analyse von Komposita (zentral auch für Bühler 1934: 320-342), wo die Form zusammengesetzt, das Gemeinte oder Genannte aber konventionell „einfach“ konzipiert ist. Hempel (1980: 29-33, 152-173) analysiert die Komposition als Übergangserscheinung zwischen ad hoc sinnsetzenden Akten der Rede

30 „Generativ“ hier natürlich nicht im Sinne bedeutungsfreier kategorialer Schemata wie in den frühen Phasen der Chomsky-Grammatik, sondern im Sinne des synsemantischen Aufbaus noetischer Gebilde.

31 Henckmann (1988: 389) behauptet sogar, Gomperz habe der sprachlichen Form gar keine „,bedeutungsaffizierende“ Funktion zugemessen. Genauer in diesen Dingen war Marty (1908, 1925).

32 Die kombinatorischen Voraussetzungen der Noetik reflektiert Gomperz (1929: 196 ff., 206 ff.) als letztlich gewohnheits- und erwartungsbasiert. 
und etablierten Bedeutungsbeständen. Durch Lexikalisierung gehen prädikative Setzungen in das Feld der Namensbestände über.

Das Tragische an der Geschichte ist, dass aus den hoch differenzierten Ansätzen zu einer Noetik, einer Lehre von dem (übereinzelsprachlich und doch sprach- und kulturspezifisch) Meinbaren und Gemeinten, am Ende (vermittels des einflussreichen Werks von Ogden \& Richards 1923) eine schlichte Referenzsemantik geworden ist, eine Lehre, in der die Aufgabe der einzelsprachlichen Konzepte (oder Sprachinhalte) ausschließlich darin zu bestehen scheint, dem Hörer den Weg zu den außersprachlichen Sachen zu bahnen, eine Lehre, in der Bedeutung als spracheigenes, sprachsystemisches noetisches Potential und fallweise Bezeichnungsleistung bis zur Ununterscheidbarkeit verschwimmen - kurz: ein Modell, in dem zusammenwächst, was durchaus nicht zusammengehört. Wer mit Kibrik (2011) das jüngste (zweifellos verdienstreiche!) Lehrbuch und Standardwerk zur Referenz aufschlägt, der fragt sich alsbald bang, wie all diese Referenten mit einem Male in das Kurzzeitgedächtnis des Sprechers gelangt sind, wo sie dann vermittels referential devices adressiert werden können. Bei aller Differenz im Detail: axiomatisch bringt uns der moderne individualistische Kognitivismus wieder genau in die Lage, aus der Husserl und Gomperz uns herausführen wollten. Hat er doch einfach nur die realen Personen und Sachen der Außenwelt, mit denen uns der Behaviorismus erden wollte, wieder in Bewusstseinsinhalte zurückverwandelt. Dass Semantik jetzt wieder der Psychologie zugeschlagen wird, dass sie zeitweise der Kulturanthropologie, zeitweise der Logik überwiesen wurde, verstärkt den Eindruck, dass sie mit den Eigenmitteln der Sprachwissenschaft nicht schlüssig zu bearbeiten sei. Offenbar passt die Noetik nicht in das Beuteschema irgendeiner etablierten wissenschaftlichen Disziplin. Ausnehmen sollte man da vielleicht nur die Ethnomethodologen, die aber eigenen terminologischen Konventionen folgen (indexicality, reflexivity, accountability) und die (kein Zufall!) ebenfalls über den Husserl-Schüler Alfred Schütz auf phänomenologische Traditionen zurückgehen.

Indessen ist der Noetik-Gedanke (freilich nicht die Bezeichnung „Noetik“) wirkmächtig nur einmal auf die linguistische Bühne zurückgekehrt, in der eher logisch-monologischen Gestalt der Husserl-Version, und zwar im „Internalismus“ und Universalismus der frühen Chomskyaner und ihrer Syntaxkonzeption. Hinter der Definition einer natürlichen Sprache L als Menge der grammatisch korrekten Sätze bzw. als kategorialer Regelapparat, mit dessen Hilfe diese Menge generiert werden kann, verbirgt sich subkutan, aber ganz unverhohlen die Gleichung: grammatischer Satz = noetisch interpretierbarer Satz. Und für den „Internalismus" der generativen Grammatik ist Referenz im Sinne der kontingenten, lokalempirischen Verweisung auf außersprachliche Gegenstände ein reines Performanzproblem, das mit der Symbolarchitektur der Sprache nur sehr locker zusammenhängt. „Standard theories of reference“, so schreibt Chomsky (1995: 236) sind auf natürliche Sprachen nicht anwendbar, schon darum nicht, weil ein und dieselbe Nominalgruppe (NP) gleichzeitig auf Abstraktes und Konkretes Bezug nehmen kann:

The book I'm writing will weigh five pounds. 
So lautet sein Beispielsatz. Das ist ein noetisches Argument. Ganz wie Husserl und Gomperz errichtet Chomsky eine strikte und unüberwindliche Barriere zwischen der Referenz (als Performanzphänomen) und den internen Symbolen, die ja in der Tat nicht referieren (Chomsky 2010: 57). Dadurch wird language „virtually synonymous with symbolic thought“ (ibid.: 59). Dass man sie auch externalisieren und zur Kommunikation mit anderen, zur wechselseitigen Orientierung in der Handlungswelt gebrauchen kann, wird dabei zum Epiphänomen, und man darf sich natürlich fragen, ob Gomperz und der späte Husserl (mit ihrer Akzentuierung der „Geltung für andere") da nicht einen Schritt weiter waren, bildlich gesprochen.

Referenz im herkömmlichen Sinne eines geteilten, vom Sprecher dem Hörer vermittelten Bezugs auf einen definiten außersprachlichen Gegenstand ist ein modellbildender Grenz- und Sonderfall geteilter sprachlicher Bezüge. Modellbildend insofern, als sich der sozial-institutionelle Charakter geteilter Bedeutungen an diesem Sonderfall etabliert. Modellbildend auch insofern, als sich unsere alltagstheoretische linguistic ideology (Silverstein 1979) an der Bezeichnungsfunktion des Sprechens orientiert. Geteilte Bedeutungen entstehen über Akte der kommunikativen Aufmerksamkeitskoordination, die in der geteilten Wahrnehmung und im Fortgang der Handlungskoordination bündig werden. Auf diesem Fundament wird die Welt der Noetik errichtet. Die Sprachwissenschaft hätte allen Grund, sie zu erkunden. Der Ausdruck der Referent hat nämlich keinen Referenten - wohl aber ein explizierbares thingmeant, das seinerseits als Zentrum möglicher Ausgiebigkeit dient. Der Referent ist insofern ein noetischer Gegenstand.

Wer moderne Lehrbücher der Semantik aufschlägt (von Lyons 1977 bis Löbner 2003), der stößt auf folgenden Befund: Wiewohl bereits Lyons (1977: 176 und öfter) davor warnt, reference als „utterance-dependent notion“ mit denotation in der Mill-Tradition gleichzusetzen oder engzuführen (denotation ist nämlich gerade als Relation zwischen einem lexikalischen Ausdruck und der extensionalen Klasse von Individuen konzipiert, die mit dem Lexem bezeichnet werden können, ist also gerade nicht äußerungsbezogen), ist eben diese Gleichsetzung bzw. Engführung die herrschende Praxis:

Die deskriptive Bedeutung eines Inhaltswortes ist ein Konzept für seine potenziellen Referenten. (Löbner 2003: 29)

Es ist aber ganz leicht zu zeigen, dass die referentielle Identifikation eines Objektes für die Aufmerksamkeit unabhängig ist von der Frage, ob die sprachliche Konzeptualisierung des Referenten auf diesen zutrifft oder nicht (vgl. Lyons 1977: 182). Wenn A sagt: Siehst du da hinten den Fuchs? Und B antwortet: Das ist ein Hund!, müssen wir voraussetzen, dass die referentielle Identifikation des Gegenstandes erfolgreich stattgefunden hat, wiewohl die sprachliche Konzeptualisierung nicht zutrifft. Die demonstrative Erfüllung eines Nennausdrucks „schlägt“ im Gebrauch dessen typisierenden Gehalt. Dagegen „schlägt“ umgekehrt die Konzeptualisierung die Identifikation, wenn letztere bereits anderweitig erfolgt ist: Wenn erkennbar von meinem Nachbarn die Rede ist, dann 
kann ich auch diesen als Hund charakterisieren. Ganz abgesehen davon, dass man referentiell auch mit Ausdrücken identifizieren kann, die auf buchstäblich jeden zutreffen können wie $i c h$ oder $d u$. Konzeptualisieren/Bezeichnen und referentiell Identifizieren sind voneinander unabhängige Teilhandlungen des Sprechens, auch wenn ihre Engführung die Regel ist.

Und für den Satz als Proposition entwirft Löbner (2003: 185) eine Version des semiotischen Dreiecks, in der ein gegebener Satz (an der Spitze des Dreiecks) seine Proposition bedeutet, über seine Referenzsituation prädiziert und in dem die komplexe Prädikation auf die Referenzsituation zutrifft. Ich reproduziere das Dreieck im Anhang zu diesem Text. Auch diese Fassung reduziert die eigensinnige Welt der Noetik letztlich auf Referenz und fällt hinter Gomperz und Husserl zurück ${ }^{33}$.

\section{LITERATUR}

Albano Leoni, Federico. 2015. Les parties et le tout: Jakobson, Husserl et la phonologie. Histoire Épistémologie Langage 37(1): 27-42.

Bréal, Michel. 1868. Les idées latentes du langage. Leçon faite au Collège de France pour la réouverture du cours de grammaire comparée le 7 décembre 1868. Paris: Librairie de L. Hachette et $\mathrm{C}^{\mathrm{ie}}$.

Bréal, Michel. 1897. Essai de sémantique (science des significations). Paris: Hachette.

Bühler, Karl. 1927. Die Krise der Psychologie. Jena: Fischer.

Bühler, Karl. 1933. Ausdruckstheorie. Das System an der Geschichte aufgezeigt. Jena: G. Fischer

Bühler, Karl. 1934. Sprachtheorie. Die Darstellungsfunktion der Sprache. Jena: G. Fischer.

Chomsky, Noam. 1995. The Minimalist Program. Cambridge, Mass. \& London: MIT Press.

Chomsky, Noam. 2010. Some Simple Evo Devo Theses: How true might they be for language? The Evolution of Human Language. Biolinguistic Perspectives, ed. by Richard K. Larson, Viviane Déprez \& Hiroko Yamakido. Cambridge, Mass.: Cambridge University Press. 45-62.

Clark, Herbert H. \& Deanna Wilkes-Gibbs. 1986. Referring as a Collaborative Process. Cognition 22(1). 1-39.

Dittrich, Ottmar. 1913. Die Probleme der Sprachpsychologie und ihre gegenwärtigen Lösungsmöglichkeiten. Leipzig: Quelle \& Meyer.

Erdmann, Karl Otto. 1922 [1900]. Die Bedeutung des Wortes. 3. Aufl. Leipzig: H. Haessel.

33 Keinesfalls würden die Anhänger Husserls in der Semantik (wie Porzig 1950; Nehring 1963) eine Proposition in der bezeichneten Referenzsituation enden oder sie dort „zutreffen“ lassen. Das würde wohl für eine epistemologische Entgleisung gelten. Für Porzig (1950: 357) haben wir es mit einer aktiven Gliederung, einer bestimmenden Extraktion sprachlicher Sachverhalte aus der außersprachlichen Realität zu tun. Nehring (1963: 161) spricht von einer Hinweisfunktion des (gesprochenen) Satzes gegenüber dem, worauf er bezogen werden kann und nähert sich damit dem Gedanken der grundsätzlichen Indexikalität alles Sprechens. 
Fehr, Johannes. 1997. Ferdinand de Saussure. Linguistik und Semiologie. Notizen aus dem Nachlass. Frankfurt a. M.: Suhrkamp.

Fleck, Ludwik. 1980 [1935]. Entstehung und Entwicklung einer wissenschaftichen Tatsache: Einführung in die Lehre vom Denkstil und Denkkollektiv. Neuausgabe mit einer Einleitung von Lothar Schäfer \& Thomas Schnelle. Frankfurt a. M.: Suhrkamp [Originalausgabe in Basel: Benno Schwabe. 1935].

Fréchette, Guillaume. 2018. Der Ausgangsgegenstand der Psychologie. Bühler und die Schule Brentanos. Karl Bühlers Krise der Psychologie. Positionen, Bezüge und Kontroversen im Wien der 1920er/30er Jahre, hrsg. von Janette Friedrich. Cham: Springer (Veröffentlichungen des Instituts Wiener Kreis, 26). 99-115.

Freyer, Hans. 1923. Theorie des objektiven Geistes. Eine Einleitung in die Kulturphilosophie. Leipzig \& Berlin: Teubner.

Friedrich, Janette. 2010. Das Erleben von Ausdruck - Einfühlung oder Zeichen? Zu Bühlers Ausdruckstheorie. Gefühl, Geste, Gesicht. Zur Phänomenologie des Ausdrucks, hrsg. von Michael Großheim \& Stefan Volke. Freiburg: Karl Alber (Neue Phänomenologie, 13). 88-113.

Gardiner, Alan H. 1951 [1932]. The Theory of Speech and Language. Oxford: Clarendon Press.

Garfinkel, Harold. 2006 [1948]. Seeing Sociologically. The Routine Grounds of Social Action, hrsg. und mit einer Einleitung von Anne Warfield Rawls. Manuskript von 1948, veröffentlicht 2006. Boulder \& London: Paradigm Publishers.

Garrod, Simon. 2011. Referential Processing in Monologue and Dialogue with and without Access to Real-World Referents. The Processing and Acquisition of Reference, ed. by Edward A. Gibson \& Neal J. Pearlmutter. Cambridge, Mass. \& London: MIT Press. 273-294.

Gomperz, Heinrich. 1908. Weltanschauungslehre: Ein Versuch die Hauptprobleme der allgemeinen theoretischen Philosophie geschichtlich zu entwickeln und sachlich zu bearbeiten, Bd. 2: Noologie, erste Hälfte: Einleitung und Semasiologie. Jena \& Leipzig: Diederichs.

Gomperz, Heinrich. 1929. Über Sinn und Sinngebilde. Verstehen und Erklären. Tübingen: J. C. B. Mohr.

Gomperz, Heinrich. 1953 [1941]. The Meanings of "Meaning". Philosophical Studies, ed. by Daniel Summer Robinson. Boston: Christopher. 251-274 [Originalausgabe in Philosophy of Science 8(2). 1941. 157-183].

Gomperz, Heinrich. 1953. Philosophical Studies, ed. by Daniel Summer Robinson. Boston: Christopher.

Gordon, W. Terrence. 1994. C. K. Ogden and Linguistics. With a new critical edition of The Meaning of Meaning. London: Routledge.

Hempel, Heinrich. 1980. Bedeutungslehre und allgemeine Sprachwissenschaft. Sprachtheoretisch-linguistische Arbeiten 1952-1973. Tübingen: Narr.

Henckmann, Wolfhart. 1988. Bewusstsein und Realität bei Külpe und Gomperz: Zwei Alternativen in der philosophischen Grundlegung der Semasiologie. Zeitschrift für Semiotik 10(4): 377-397.

Holenstein, Elmar. 1975. Roman Jakobsons phänomenologischer Strukturalismus. Frankfurt a. M.: Suhrkamp. 
Husserl, Edmund. 1913 [1901]. Logische Untersuchungen, Bd. II, Teil 1: Untersuchungen zur Phänomenologie und Theorie der Erkenntnis. 2. Aufl. Tübingen: Niemeyer.

Husserl, Edmund. 1948 [1938]. Erfahrung und Urteil, hrsg. von Ludwig Landgrebe. Hamburg: Claasen \& Goverts.

Kibrik, Andrej A. 2011. Reference in Discourse. Oxford: Oxford University Press.

Kiesow, Karl-Friedrich. 1988. Aussageinhalt bei Gomperz, Bühler und Popper. Karl Bühler's Theory of Language, hrsg. von Achim Eschbach. Amsterdam, Philadelphia: John Benjamins. 349-368.

Knobloch, Clemens. 1988. Geschichte der psychologischen Sprachauffassung in Deutschland zwischen 1850 und 1920. Tübingen: Niemeyer.

Knobloch, Clemens. 2020. Von der Bedeutung zum Gemeinten (und zurück): Semantik und Noetik. Sine ira et studio. Disziplinenübergreifende Annäherungen an die zwischenmenschliche Kommunikation, hrsg. von Robin Kurilla, Karin Kolb-Albers, Hannes Krämer \& Karola Pitsch. Heidelberg: Springer. 11-35.

Koschmieder, Erwin. 1965. Beiträge zur allgemeinen Syntax. Heidelberg: Winter.

Löbner, Sebastian. 2003. Semantik. Eine Einführung. Berlin \& New York: De Gruyter.

Lübbe, Hermann. 1960. Positivismus und Phänomenologie. Mach und Husserl. Beiträge zu Philosophie und Wissenschaft. Wilhelm Szilasi zum 70. Geburtstag, hrsg. von H. Höfling. München: Francke. 161-185.

Lyons, John. 1977. Semantics, Bd. I. London \& Cambridge: Cambridge University Press.

Maas, Utz. 2016. Sprachforschung in der Zeit des Nationalsozialismus. Berlin \& Boston: De Gruyter.

Maas, Utz. 2018. Heinrich Gomperz. Verfolgung und Auswanderung deutschsprachiger Sprachforscher 1933-1945. Berlin: Zentrum für Literatur- und Kulturforschung [https://zflprojekte.de/sprachforscher-im-exil/index.php/catalog/g/519 -gomperz, zuletzt eingesehen 15.1.2021].

Maas, Utz. 2019. Karl Mannheim. Verfolgung und Auswanderung deutschsprachiger Sprachforscher 1933-1945. Berlin: Zentrum für Literatur- und Kulturforschung [https://zflprojekte.de/sprachforscher-im-exil/index.php/catalog/m/535-mannhei m,-karl, zuletzt eingesehen 15.1.2021].

Maas, Utz. 2020. Edmund Husserl. Verfolgung und Auswanderung deutschsprachiger Sprachforscher 1933-1945. Berlin: Zentrum für Literatur- und Kulturforschung [https://zflprojekte.de/sprachforscher-im-exil/index.php/catalog/h/268-husserledmund, zuletzt eingesehen 15.1.2021].

Mangold-Allwinn, Roland, Stefan Barattelli, Markus Kiefer \& Hanns Gerhard Koelbing, Hrsg. 1995. Wörter für Dinge. Von flexiblen Konzepten zu variablen Benennungen. Opladen: Westdeutscher Verlag.

Mannheim, Karl. 1980 [1924]. Eine soziologische Theorie der Kultur und ihrer Erkennbarkeit (konjunktives und kommunikatives Denken). Strukturen des Denkens, hrsg. von David Kettler, Volker Meja \& Nico Stehr. Frankfurt a. M.: Suhrkamp. 155-322. 
Marty, Anton. 1908. Untersuchungen zur Grundlegung der allgemeinen Grammatik und Sprachphilosophie. Halle a. S.: Niemeyer.

Marty, Anton. 1925. Satz und Wort. Eine kritische Auseinandersetzung mit der üblichen grammatischen Lehre und ihren Begriffsbestimmungen. Aus dem Nachlass herausgegeben von Otto Funke. Reichenberg: Stiepel.

McElvenny, James. 2014. Ogden and Richards' The Meaning of Meaning and Early Analytical Philosophy. Language Sciences 41: 212-221.

McElvenny, James. 2018. Language and Meaning in the Age of Modernism: C. K. Ogden and his Contemporaries. Edinburgh: Edinburgh University Press [zugleich: Diss. Phil. Sydney: University of Sydney. 2013].

Nehring, Alfons. 1963. Sprachzeichen und Sprechakte. Heidelberg: Winter.

Nerlich, Brigitte. 1992. Semantic Theories in Europe, 1830-1930. Amsterdam: John Benjamins.

Ogden, Charles K. \& Ivor A. Richards. 1923. The Meaning of Meaning: A study of the influence of language upon thought and of the science of symbolism. London: Routledge \& Kegan Paul.

Ogden, Charles K. \& Ivor A. Richards. 1974 [1923]. Die Bedeutung der Bedeutung. Frankfurt a. M.: Suhrkamp [Übersetzung von: The Meaning of Meaning. 1923. London: Routledge \& Kegan Paul. 10. Aufl. 1949].

Porzig, Walter. 1950. Das Wunder der Sprache. Bern: A. Francke.

Ritter, Joachim \& Karlfried Gründer. 1984. Historisches Wörterbuch der Philosophie, Bd. 6. Basel \& Stuttgart: Schwabe.

Romand, David. 2019. More on Formal Feeling/Form-Feeling in Language Sciences. Heinrich Gomperz's Concept of "Formal Logical Feeling” (logisches Formalgefühl) Revisited. Histoire Épistémologie Langage 41(1): 131-157.

Sandmann, Manfred. 1940. Substantiv, Adjektiv-Adverb und Verb als sprachliche Formen. Bemerkungen zur Theorie der Wortarten. Indogermanische Forschungen 57: 81-112.

Sandmann, Manfred. 1954. Subject and Predicate. A Contribution to the Theory of Syntax. Edinburgh: Edinburgh University Press.

Sapir, Edward. 1927. The Unconscious Patterning of Behavior in Society. The Unconscious. A Symposium, hrsg. von E. S. Dummer. New York: Knopf. 114-142.

Sapir, Edward. 1931. Communication. Encyclopedia of the Social Sciences 4: 78-81.

Seiler, Martin \& Friedrich Stadler, Hrsg. 1994. Heinrich Gomperz, Karl Popper und die österreichische Philosophie. Beiträge zum internationalen Forschungsgespräch des Instituts „Wiener Kreis“ aus Anlass des 50. Todestages von Heinrich Gomperz und des 90. Geburtstages von Karl Popper, Wien, 1992. Amsterdam \& Atlanta, Georg.: Rodopi.

Silverstein, Michael. 1979. Language Structure and Linguistic Ideology. The Elements: A Parasession on Linguistic Units and Levels, hrsg. von Paul R. Clyne, William F. Hanks \& Carol L. Hofbauer. Chicago: Chicago Linguistic Society. 193-247.

Sperber, Dan \& Deirdre Wilson. 1986. Relevance: Communication and Cognition. Oxford: Blackwell. 
Trueswell, John C., Anna Papafragou \& Youngon Choi. 2011. Referential and Syntactic Processes: What Develops? The Processing and Acquisition of Reference, ed. by Edward A. Gibson \& Neal J. Pearlmutter. Cambridge, Mass. \& London: MIT Press. 65-108.

Wegener, Philipp. 1885. Untersuchungen über die Grundfragen des Sprachlebens. Halle a. S.: Niemeyer.

Wittgenstein, Ludwig. 1971 [1922]. Tractatus logico-philosophicus. Frankfurt a. M.: Suhrkamp.

Wittgenstein, Ludwig. 1973. Philosophische Grammatik. Frankfurt a. M.: Suhrkamp. Znaniecki, Florian. 1919. Cultural Reality. Chicago: Chicago University Press.

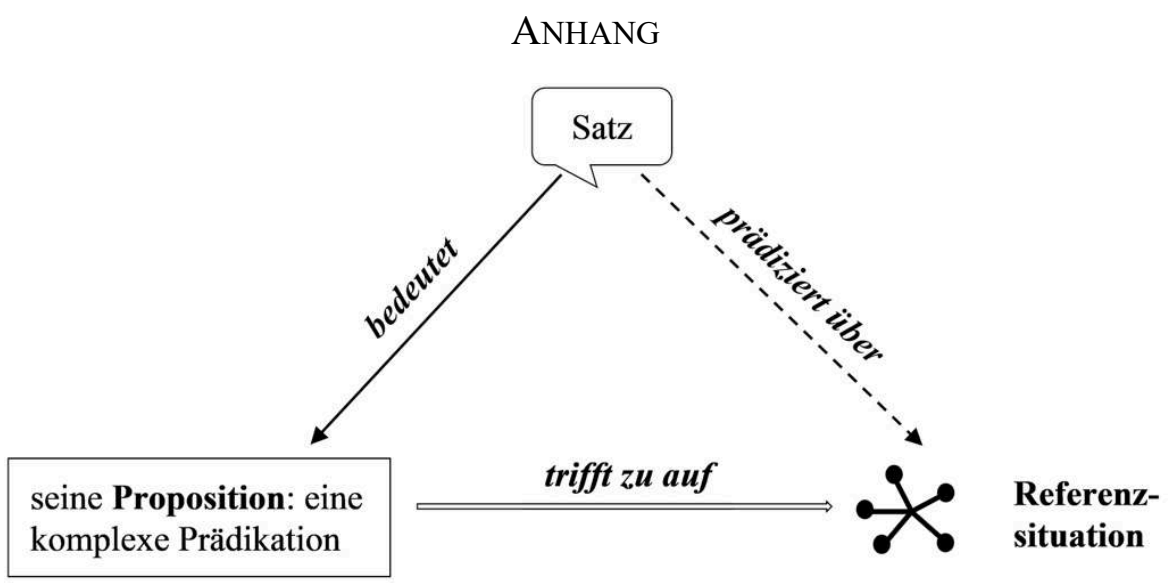

Abbildung 2: Das semiotische Dreieck für den Satz als Prädikation (adaptiert von Löbner 2003: 185). 
